\title{
A Model of the Block of Voltage-Gated Potassium Kv4.2 Ionic Currents by 4-Aminopyridine
}

\author{
Steven J. Kehl \\ Department of Cellular and Physiological Sciences, University of British Columbia, Vancouver, British Columbia, Canada \\ Received May 25, 2017; accepted August 28, 2017
}

\begin{abstract}
Voltage clamp recordings of macroscopic currents were made from rat potassium-gated potassium 4.2(Kv4.2) channels expressed in human embryonic kidney (HEK293) cells with the main goals of quantifying the concentration, time, and voltage dependence of the block and to generate a state model that replicates the features of the block. When applied either externally or internally, the block of Kv4.2 currents by 4-aminopyridine (4AP) occurs at the holding potential $(-80 \mathrm{mV})$, is affected by the stimulus frequency, and is relieved by membrane depolarization. The $K_{d}$ for the tonic block at $-80 \mathrm{mV}$ was $0.9 \pm 0.07 \mathrm{mM}$ and was consistent with $1: 1$ binding.
\end{abstract}

Relief of block during a step to $50 \mathrm{mV}$ was well fitted by a single exponential with a time constant of $\sim 40$ milliseconds. At $-80 \mathrm{mV}$, the association rate constant was $0.08 \mathrm{mM}^{-1} \mathrm{~s}^{-1}$, and the off-rate was $0.08 \mathrm{~s}^{-1}$. The state model replicates the features of the experimental data reasonably well by assuming that 4AP binds only to closed states, that 4AP binding and inactivation are mutually exclusive processes, and that the activation of closedbound channels is the same as for closed channels. Since the open channel has a very low or no affinity for 4AP, channel opening promotes the unbinding of $4 \mathrm{AP}$, which accounts for the reverse use dependence of the block.

\section{Introduction}

Investigations of the mechanisms by which organic compounds affect ionic and gating currents mediated by voltagegated channels have played an important role in developing our understanding of the molecular structure of ion channels. In the context of voltage-gated $\mathrm{K}^{+}\left(\mathrm{K}_{\mathrm{v}}\right)$ channels, the seminal studies by Armstrong (1971) of the block of delayed rectifier $\mathrm{K}^{+}$ channels of the squid giant axon (SGA) by quaternary ammonium compounds, such as tetraethylammonium ion $\left(\mathrm{TEA}^{+}\right)$, have provided insights into the topology of the permeation pathway, as well as the location and movement of the activation gate. Aminopyridines comprise another group of compounds that have been useful tools in the characterization of $\mathrm{Kv}$ channels. For example, 4-aminopyridine (4AP) was first reported to block axonal delayed rectifier currents from several invertebrate and vertebrate species (Yeh et al., 1976b; Kirsch and Narahashi, 1983) and more recently to block currents mediated by the fast inactivation-removed Shaker (ShIR) channel (Castle et al., 1994a; McCormack et al., 1994), which is the Drosophila homolog of the Kv1 channel subfamily.

It has been proposed for Shaker channels (Armstrong and Loboda, 2001) that both $\mathrm{TEA}^{+}$and $4 \mathrm{AP}$ have a site of action in the inner vestibule of the pore (Armstrong, 1997) that is nominally equivalent to the central cavity identified in the

Supported by a Discovery Grant to S.K. from the Natural Sciences and Engineering Research Council of Canada.

http://doi.org/10.1124/jpet.117.243097. crystal structure of the KcsA channel, a potassium channel isolated from Streptomyces lividans (Doyle et al., 1998). For both compounds, the experimental data are consistent with the view that the access of internal 4AP (Kirsch and Drewe, 1993; McCormack et al., 1994) to a binding site in the central cavity is contingent on opening of the activation gate that otherwise prevents movement of compounds from the cytoplasmic compartment into the central cavity and vice versa in the case of unbinding. A consequence of this strict coupling of binding to channel opening is that the block is use-dependent, meaning that membrane depolarization is required to initiate the block. The primary distinction between TEA ${ }^{+}$and $4 \mathrm{AP}$ is the outcome of binding in the central cavity. In the case of $\mathrm{TEA}^{+}$, which is approximately the size of a hydrated $\mathrm{K}^{+}$ion, the inhibition of current comes about by a physical plugging of the permeation pathway. In the case of $4 \mathrm{AP}$, which is smaller than $\mathrm{TEA}^{+}$, binding in the central cavity of the Shaker channel is proposed to strongly bias the opening/closing transitions toward the closed state, thus decreasing the open probability $\left(P_{o}\right)$. The latter effect is associated with no or minimal alteration of the kinetics or voltage dependence of voltage-sensor activation (McCormack et al., 1994; Loboda and Armstrong, 2001).

Fast-activating, fast-inactivating $\mathrm{K}^{+}$current, which is identified as transient outward current $\left(I_{t o}\right)$ in cardiac myocytes and as $I_{A}$ in central neurons and is mediated by $\mathrm{Kv} 4$ (Shal) channels (Fiset et al., 1997), is also blocked by 4AP. As first noted in the study by Thompson (1977), the properties of

ABBREVIATIONS: 4AP, 4-aminopyridine; CSI, closed-state inactivation; HEK293, human embryonic kidney cell line 293; $I_{\text {to }}$, transient outward current; $k_{\text {off }}$, unbinding rate constant; $k_{o n}$, association rate constant; $\mathrm{Kv}$, voltage-gated potassium; $P_{\mathrm{o}}$, open probability; $s$, slope factor; SGA, squid giant axon; ShIR, fast inactivation removed Shaker $\mathrm{K}^{+}$channel; $\tau_{\text {unblock}}$, time constant for unblock; $\tau_{\text {block }}$, time constant for block; $\mathrm{TEA}^{+}$, tetraethylammonium ion; $V_{h}$, holding potential; $V_{\text {half }}$, voltage for half-maximal effect. 
the block of $I_{A}$ in molluscan central neurons showed some similarities to its block of the SGA delayed rectifier (Yeh et al., 1976a). Thus, 4AP block increases with hyperpolarization, is use-dependent, and can be removed by depolarization. The block of $I_{t o}$ in ventricular myocytes (Campbell et al., 1993; Castle and Slawsky, 1993) of the transient $\mathrm{K}^{+}$current $\left[I_{K}(f)\right]$ in pituitary melanotrophs (Kehl, 1990) and of Kv4.2 channels in a heterologous expression system (Tseng et al., 1996; Yeola and Snyders, 1997) suggested that 4AP binding and inactivation were mutually exclusive events; that is to say, inactivation was prevented in 4AP-bound channels, and 4AP binding was prevented by inactivation. Additionally, binding of 4AP occurred exclusively to one or more closed states, which gives rise to the phenomenon of "reverse use-dependence" (Tseng et al., 1996) and distinguishes the 4AP block of transient $\mathrm{K}^{+}$ currents from the block of Shaker channels.

To address the issue of the mechanism of action of 4AP in fast transient $\mathrm{K}^{+}$channels, this study set two goals. The first was to describe the inhibition by $4 \mathrm{AP}$ of $\mathrm{Kv} 4.2$ channels expressed in HEK293 cells and to quantify the concentration, time, and voltage dependence of the block. The second goal was to use the quantitative measures gleaned from the experimental data to constrain a numerical model of the actions of 4AP. The proposed model replicates the features of the experimental data reasonably well by assuming that $4 \mathrm{AP}$ binds to closed states, that $4 \mathrm{AP}$ binding and inactivation are mutually exclusive processes, and that the activation of closed-bound channels is the same as for closed channels. By virtue of a low or no affinity of the open channel for 4AP, channel opening promotes the unbinding of 4AP, which accounts for the reverse use dependence of the block.

\section{Materials and Methods}

Cell Preparation. Currents were recorded from rat $\mathrm{Kv} 4.2$ channels stably expressed in a human embryonic kidney cell line (HEK293; American Type Culture Collection, Rockville, MD). Cells were passaged using trypsin-EDTA and maintained in minimum essential medium (MEM) supplemented with $10 \%$ fetal bovine serum, $1 \%$ penicillin-streptomycin, and $0.5 \mathrm{mg} \mathrm{ml}^{-1}$ geneticin at $37^{\circ} \mathrm{C}$ in an atmosphere of $5 \% \mathrm{CO}_{2}$ in air. All tissue culture supplies were obtained from Invitrogen (Burlington, ON, Canada).

Recording Solutions. Unless otherwise stated, the standard bath solution contained (in millimolars) the following: $140 \mathrm{NaCl}, 3.5$ $\mathrm{KCl}, 2 \mathrm{CaCl}_{2}, 1 \mathrm{MgCl}_{2}, 5$ glucose, and 10 HEPES; the $\mathrm{pH}$ was adjusted with $\mathrm{NaOH}$ to 7.4 at room temperature. A stock solution of $4 \mathrm{AP}$ for bath application was prepared by dissolving 4-aminopyridine (SigmaAldrich Chemical Company, $\geq 99 \%$; ) in standard bath solution and then titrating to $\mathrm{pH} 7.4$ with $\mathrm{HCl}$. The patch pipette solution contained (in millimolars) the following: $130 \mathrm{KCl}, 4.75 \mathrm{CaCl}_{2}(p \mathrm{Ca}=7.3), 1.38$ $\mathrm{MgCl}_{2}, 10$ EGTA, and 10 HEPES and was adjusted to $\mathrm{pH} 7.4$ with potassium hydroxide. For experiments assessing the effects of its internal application, a $20 \mathrm{mM}$ stock solution of $4 \mathrm{AP}$ was prepared in the patch pipette solution and then titrated to $\mathrm{pH} 7.4$ with $\mathrm{HCl}$. Chemicals were obtained from the Sigma-Aldrich Chemical Company.

A section of glass coverslip to which stably transfected HEK293 cells had adhered was placed in a recording chamber ( 0.5 -ml volume). During this experiment, the chamber was perfused with standard bath solution at a rate of $1-2 \mathrm{ml} \mathrm{min}^{-1}$. External 4 AP was applied using a fast-application pipette positioned within $100 \mu \mathrm{m}$ of the test cell, and rapid change of the pipette solution perfusing a test cell was digitally controlled (Cheng et al., 2010).

Electrophysiological Procedures. Whole-cell currents were recorded at room temperature $\left(20-25^{\circ} \mathrm{C}\right)$ using an EPC-8 patch clamp amplifier connected to a computer via an ITC-18 digital interface (HEKA Electronik, Lambrecht, Germany). Patch electrodes were made from thinwalled borosilicate glass (World Precision Instruments, Sarasota, FL) and had resistances of 1.0-1.5 $\mathrm{M} \Omega$ measured in the recording chamber with the standard internal and external solutions. Voltage clamp commands were generated using HEKA PatchMaster software. Circuitry of the EPC-8 amplifier was used to compensate the membrane capacitance and at least $80 \%$ of the series resistance. Leak subtraction was not used. Current signals were filtered at $3 \mathrm{kHz}$ ( $-3 \mathrm{~dB}$, eight-pole Bessel filter) and digitized (16-bit resolution) at a sampling interval of 25-50 microseconds. Voltages were corrected for liquid junction potentials. The holding potential $\left(V_{h}\right)$ was normally $-80 \mathrm{mV}$, and the test pulse potential was usually $50 \mathrm{mV}$. There was no compensation for the contribution of the endogenous, slowly inactivating, delayed rectifier-type current expressed in HEK293 cells. In nontransfected cells in standard bath solution, the endogenous $\mathrm{K}^{+}$current at $50 \mathrm{mV}$ typically ranges from 0.4 to $1.4 \mathrm{nA}$.

As reported previously (Kehl et al., 2013), control Kv4.2 currents had biophysical properties (not shown) that quite closely matched those reported for rat Kv4.2 channels expressed in Xenopus oocytes (Tseng et al., 1996) and wild-type human Kv4.2 channels expressed in HEK293 cells (Bähring et al., 2001). The midpoint of the steadystate availability curve was $-59.3 \pm 0.3 \mathrm{mV}$, and the slope factor was $5.4 \pm 0.2 \mathrm{mV}(n=9)$. Recovery from inactivation at $-80 \mathrm{mV}$ after a 300 -millisecond step to $50 \mathrm{mV}$ was monoexponential and had a time constant of $330 \pm 14$ milliseconds $(n=15)$

Data Analysis. Data are presented as the mean \pm the S.E.M., except for the values derived from nonlinear least-squares fitting routines (Igor Pro 7; Wavemetrics, Tigard, OR), which are expressed as the mean \pm S.D. The value for $n$ represents the number of cells examined. The level for statistical significance was $P<0.05$.

Concentration-response data were fitted to the Hill equation in eq. 1 :

$$
y=\text { base }+\frac{1-\text { base }}{1+\left\{\frac{[4 A P]}{K_{d}}\right\}^{h}},
$$

where $y$ is the normalized peak current evoked at a given concentration of $4 \mathrm{AP}$, base is the lower asymptote, $K_{d}$ is the apparent equilibrium dissociation constant, and $h$ is the Hill coefficient.

Exponential relaxations (eq. 2 and eq. 3 ) were fitted either to a monoexponential,

$$
y(t)=A_{0}+A_{1} \exp \left(-\frac{t}{\tau_{1}}\right),
$$

or a biexponential function,

$$
y(t)=A_{0}+A_{1} \exp \left(-\frac{t}{\tau_{1}}\right)+A_{2} \exp \left(-\frac{t}{\tau_{2}}\right),
$$

where $y$ is the current as a function of time $(t), A_{0}$ is the steady-state current, and $A_{1}$ and $A_{2}$ represent the proportion of the current fitted by an exponential with a time constant of $\tau_{1}$ and $\tau_{2}$, respectively.

Biphasic exponential relaxations, such as those observed in the time course of the recovery from inactivation and the onset of block (Fig. 4), were fitted using eq. 4:

$$
y(t)=A_{0}+A_{1}\left(1-\exp \left(-\frac{t}{\tau_{1}}\right)\right)+A_{2} \exp \left(-\frac{t}{\tau_{2}}\right),
$$

where $A_{1}$ and $A_{2}$ represent the amplitudes of the rising and decaying components of the current, respectively.

For the analysis of the effect of a conditioning or prepulse potential on the block by $4 \mathrm{AP}$ (Fig. 6), peak test current amplitudes were normalized using eq. 5 :

$$
y=\frac{I_{\max }-I_{\text {test }}}{I_{\max }-I_{\min }}
$$

where $y$ is the relative block, $I_{\max }$ is the maximal peak current following a conditioning voltage of $-30 \mathrm{mV}, I_{\text {test }}$ is the peak test 
current after a given conditioning voltage, and $I_{\min }$ is the peak test current after a conditioning voltage of $-80 \mathrm{mV}$. Curves relating the relative block as a function of the conditioning voltage $(V)$ were fitted to the Boltzmann equation (eq. 6):

$$
\text { relative block }(V)=\frac{1}{1+\exp \left(\frac{V_{\text {half }}-V}{s}\right)},
$$

where $V_{\text {half }}$ is the potential at which the block is half-maximal, and $s$, the slope factor, indicates the steepness of the change of relative block (i.e., limiting slope of $e$-fold change per $s$ millivolt).

Numerical Modeling. Macroscopic Kv4.2 currents were simulated and analyzed in Igor Pro 7 using built-in and user-generated functions and procedures. State occupancies as a function of time and voltage were calculated, as outlined by Colquhoun and Hawkes (2011), from the spectral expansion of the Q-matrix generated from the state diagram (Fig. 7). For voltage-dependent transitions, the rate constant was calculated according to: $k(\mathrm{~V})=k(0 \mathrm{mV}) \exp (z F V / R T)$, where $F, R$, and $T$ have their usual thermodynamic meaning, $k(0 \mathrm{mV})$ is the rate constant at $0 \mathrm{mV}$, and $z$ is the equivalent charge moved between that state and the transition state. For the calculation of currents, the open channel $i$ - $V$ relationship was assumed to be ohmic.

Several of the figures include simulated current traces generated from the model to facilitate a comparison with the experimental traces. A consideration of the simulated data in those figures is deferred to the end of the Results section, where details of the gating model and its output are presented.

\section{Results}

Time Course of Onset and Washout of 4AP-Induced Inhibition of Kv4.2. With $\mathrm{Kv} 4.2$ channels expressed in Xenopus oocytes (Tseng et al., 1996), the reversibility of 4AP was quite limited, which prompted an investigation of the dynamics of the onset and offset of the 4AP in Kv4.2 channels expressed in HEK293 cells. The diary plot of Fig. 1 shows the peak amplitudes of currents evoked during wash-in and washout of $1 \mathrm{mM}$, then $10 \mathrm{mM}$, followed by a repeat application of $1 \mathrm{mM} 4 \mathrm{AP}$; bracketed numbers below data points in the graph refer to current traces shown above the graph. The holding potential $\left(V_{h}\right)$ in the experiment was $-80 \mathrm{mV}$. At the start of the experiment, two control $(0 \mathrm{mM}$ $4 \mathrm{AP}$ ) currents, evoked at $50 \mathrm{mV}$ and separated by an interval of 20 seconds, had peak amplitudes of $\sim 18.8 \mathrm{nA}$. Using the rapid perfusion system, the cell was then perfused with $1 \mathrm{mM}$ $4 \mathrm{AP}$ for 60 seconds; the membrane voltage was maintained at $V_{h}$. Roughly 325 milliseconds before switching back to control medium, a 300 -millisecond voltage step to $50 \mathrm{mV}$ evoked a peak current of $10.4 \mathrm{nA}$, indicating a $45 \%$ decrease relative to the control currents. This decrease confirms that the onset the $4 \mathrm{AP}$ block is not contingent on membrane depolarization (Campbell et al., 1993; Castle and Slawsky, 1993; Tseng et al., 1996). Pulses to $50 \mathrm{mV}$ applied at $0.05 \mathrm{~Hz}$ after switching back to control medium indicated that the peak amplitude of the current had recovered to $19.0 \mathrm{nA}$ at $t=160$ seconds (i.e., 80 seconds after the changeover). At that point, a perfusion with $10 \mathrm{mM} 4 \mathrm{AP}$ began, and 40 seconds after the changeover $(t=200$ seconds $)$, the peak current had decreased by $\sim 70 \%$ to $5.6 \mathrm{nA}$. Because $4 \mathrm{AP}$ block of transient outward currents is use-dependent (see following), voltage steps were suspended for roughly 80 seconds and then restarted 325 milliseconds before returning to control solution. The amplitudes of the trace 3 and trace 4 currents were virtually the same, indicating that steady-state inhibition with $10 \mathrm{mM} 4 \mathrm{AP}$ could occur with a 20 -second cycle length. Reversal of block by $10 \mathrm{mM} 4 \mathrm{AP}$ was slower than with a $1 \mathrm{mM}$ concentration but was nonetheless complete 100 seconds after washout ( $t=380$ seconds). Finally, switching back to $1 \mathrm{mM}$ $4 \mathrm{AP}$ indicates that the onset of its block is comparable to that with $10 \mathrm{mM}$

Inspection of the current traces in Fig. 1 confirms two well established effects of $4 \mathrm{AP}$ on transient outward currents
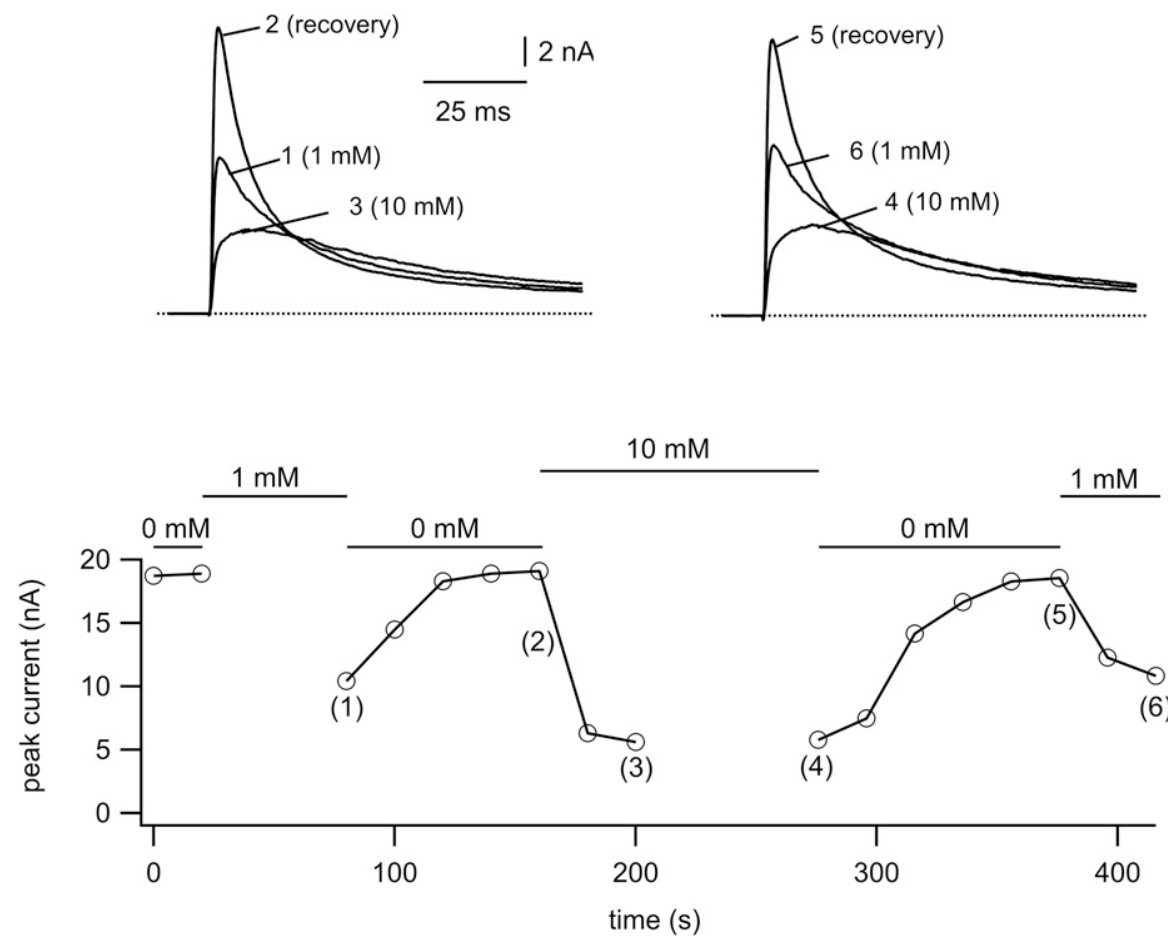

Fig. 1. A diary plot of the peak Kv 4.2 current tracking the time course of the onset and offset of externally applied 1 and $10 \mathrm{mM}$ 4AP. Solution changes and their timing are indicated by the horizontal lines in the graph. Bracketed numbers below select data points in the plot refer to the corresponding current traces above. See text for experimental details. The current traces in 1 and $10 \mathrm{mM} 4 \mathrm{AP}$ cross over the control current, and a prominent slowing of the activation is evident in $10 \mathrm{mM}$ 4AP. In these and subsequent current traces, the dotted line indicates the zero current level. 
(Thompson, 1982; Kehl, 1990; Castle and Slawsky, 1993; Tseng et al., 1996; Yeola and Snyders, 1997). First, the rise time of the current in the presence of the blocker is slowed, which is better seen in the traces evoked in $10 \mathrm{mM} 4 \mathrm{AP}$. Second, the decay of the current in 4AP is slowed. These two effects cause a crossover of the control and treated currents.

Steady-State Concentration-Response Relationship. The concentration dependence of the steady-state block of Kv4.2 was determined with the awareness that the 4AP block of transient outward currents decreases, within limits, as the stimulus frequency increases. Consequently, the approach was to record control responses and then to switch to a known concentration of $4 \mathrm{AP}$ in the absence of any depolarizing pulses $\left(V_{h}=-80 \mathrm{mV}\right)$ for a period of 60 seconds with 4AP concentrations less than $2 \mathrm{mM}$ and for 40 seconds with $2 \mathrm{mM}$ and higher concentrations. After this equilibration period, four test voltage steps (P1-P4) to $50 \mathrm{mV}$, each lasting for 300 milliseconds, were applied at a frequency of $0.8 \mathrm{~Hz}$ (cycle length, 1.25 seconds). A representative example of one such experiment is provided in Fig. 5A, which shows, superimposed, a control current as well as the test currents in $20 \mathrm{mM}$ 4AP-evoked by pulses $1-4$. Note the slow rise time of the $\mathrm{P} 1$ current and that during the decay phase it crosses over the control current.

This section is restricted to a consideration of the effect of the 4AP concentration on the amplitude of the first of the four test pulses, which is taken as a measure of the steady-state or tonic block. A consideration of the basis for the increase of the current amplitude of $\mathrm{P} 2$ and subsequent pulses that is evident in Fig. 5A is deferred until some details of the kinetics of $4 \mathrm{AP}$ unbinding (Fig. 3) and binding (Fig. 4) have been considered.

For the graph of Fig. 2, the P1 test current amplitudes in $4 \mathrm{AP}$ concentrations ranging from 0.2 to $20 \mathrm{mM}$ were normalized with respect to the average of the control and recovery responses. A fit of the concentration dependence of the experimental, normalized $\mathrm{P} 1$ test currents to the Hill equation (eq. 1; solid line, Fig. 2) gave an estimate for the $K_{d}$, the concentration producing $50 \%$ of the maximal block, of $0.9 \pm$ $0.07 \mathrm{mM}$. The best fit for the lower asymptote, which represents the normalized current in the presence of a saturating concentration of $4 \mathrm{AP}$ was $0.28 \pm 0.02$. In other words, the maximum block is roughly $72 \%$. This value for the maximum block is related to the fact that current was measured at the peak and that relatively rapid unbinding of $4 \mathrm{AP}$ occurs during a step to $50 \mathrm{mV}$. The dashed line of Fig. 2 represents the best fit of the concentration-response data $(\nabla)$ generated from the gating model (Fig. 7) and will be considered in the last section of the Results.

Increasing the concentration of $4 \mathrm{AP}$ also affected the time to peak of the current, which in control cells was $2.87 \pm 0.08$ milliseconds $(n=16)$. Although in $2 \mathrm{mM} 4 \mathrm{AP}$ the effect was relatively small $(3.14 \pm 0.23, n=4)$, there was a progressive increase in the time to peak to $10.4 \pm 1.4$ milliseconds $(n=7)$ in $5 \mathrm{mM}$, to $15.9 \pm 1.2$ milliseconds $(n=11)$ in $10 \mathrm{mM}$, and to $18.6 \pm 1.1$ milliseconds $(n=3)$ in $20 \mathrm{mM}$.

The decay rate of the $\mathrm{P} 1$ test current was also concentrationdependent. In control cells, inactivation during a 300-millisecond step from -80 to $50 \mathrm{mV}$ was well fitted by a biexponential function where the faster component had a time constant $\left(\tau_{f}\right)$ of $7.8 \pm 0.2$ milliseconds and accounted for $0.75 \pm$ 0.008 ( $n=19$ ) of the total current decay and the slower component had a time constant $\left(\tau_{s}\right)$ of $40.2 \pm 1.4$ milliseconds

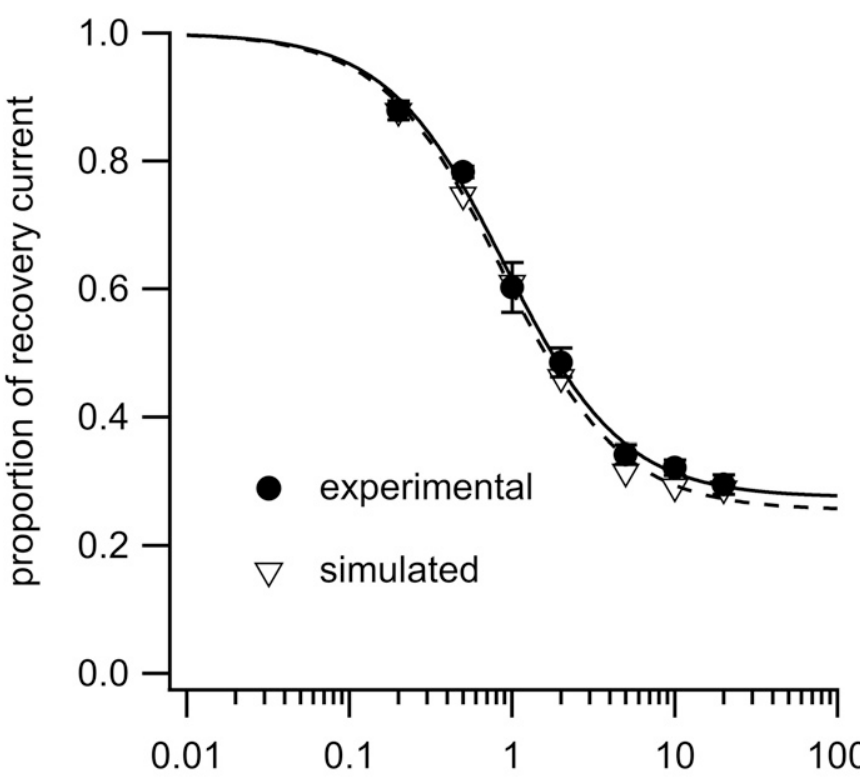

4AP concentration in $\mathrm{mM}$

Fig. 2. The experimental and simulated concentration-response relationship for the interaction of $4 \mathrm{AP}$ with $\mathrm{Kv} 4.2$ channels. Experimental data $(\bullet)$ (mean \pm S.E.M.) representing tonic block were generated from the peak current evoked by a 300 -millisecond step to $50 \mathrm{mV}$ after an interval of $60(<2 \mathrm{mM})$ or 40 seconds $(2 \mathrm{mM}$ and higher) at the holding potential of $-80 \mathrm{mV}$. The peak current, measured once the binding equilibrium was reached, was normalized to the average of the control and recovery currents. The solid line indicating the best fit of the data points to the Hill equation (eq. 1) was obtained with $K_{d}=0.9 \pm 0.07 \mathrm{mM}, h=1.2 \pm 0.07$, and base $=0.28 \pm 0.02$. Concentrationresponse data $(\nabla)$ generated from Scheme $4 \mathrm{AP}$ (Fig. 7) over the same range of $4 \mathrm{AP}$ concentrations was well fitted by the eq. 1 (dashed line) with $K_{d}=0.88 \pm$ $0.06 \mathrm{mM}, h=1.16 \pm 0.07$ and base $=0.25 \pm 0.02$.

and a relative amplitude of $0.25 \pm 0.008(n=19)$. With 4AP concentrations of $2 \mathrm{mM}$ or less, the current decay was biexponential, with the main effect appearing to be a decreased contribution of the faster component and a slower relaxation of the second component. For example, with $2 \mathrm{mM}$ $4 \mathrm{AP} \tau_{f}$ was $10.2 \pm 1.1$ milliseconds, $\tau_{s}$ was $51.9 \pm 4.5$ milliseconds, and the relative amplitudes of the two components were similar (0.473 and 0.527 , respectively; $n=5)$; however, with higher concentrations, the decay rate was well fitted by a single exponential with a time constant of $51.9 \pm 2.3$ milliseconds $(n=7)$ with $5 \mathrm{mM}, 58.1 \pm 1.9$ milliseconds $(n=10)$ with $10 \mathrm{mM}$ and $64.2 \pm 3.6(n=3)$ with $20 \mathrm{mM}$. The basis for these changes in current decay is addressed in the following section dealing with the proposed model for $4 \mathrm{AP}$ block of $\mathrm{Kv} 4.2$ currents.

4AP Unbinding from Kv4.2 Channels at $50 \mathrm{mV}$. Castle and Slawsky (1993) proposed that in $I_{t o}$ channels of rat ventricular myocytes, 4AP binds most strongly to closed states. In their study of macroscopic Kv4.2 currents Tseng et al. (1996) concluded that 4AP binds exclusively to closed states. A corollary of this state of dependence of binding is that depolarization triggers 4AP unbinding. To characterize the time dependence of the dissociation of 4AP during strong membrane depolarization, a two-pulse protocol was used (Fig. 3A). Briefly, for the first sweep, an 8-millisecond conditioning pulse to $50 \mathrm{mV}$ was used, and its duration was doubled on successive sweeps up to 512 milliseconds. To allow near complete recovery from inactivation $(\tau \approx 330$ milliseconds) but limit the time available for $4 \mathrm{AP}$ rebinding to closed states, 
A
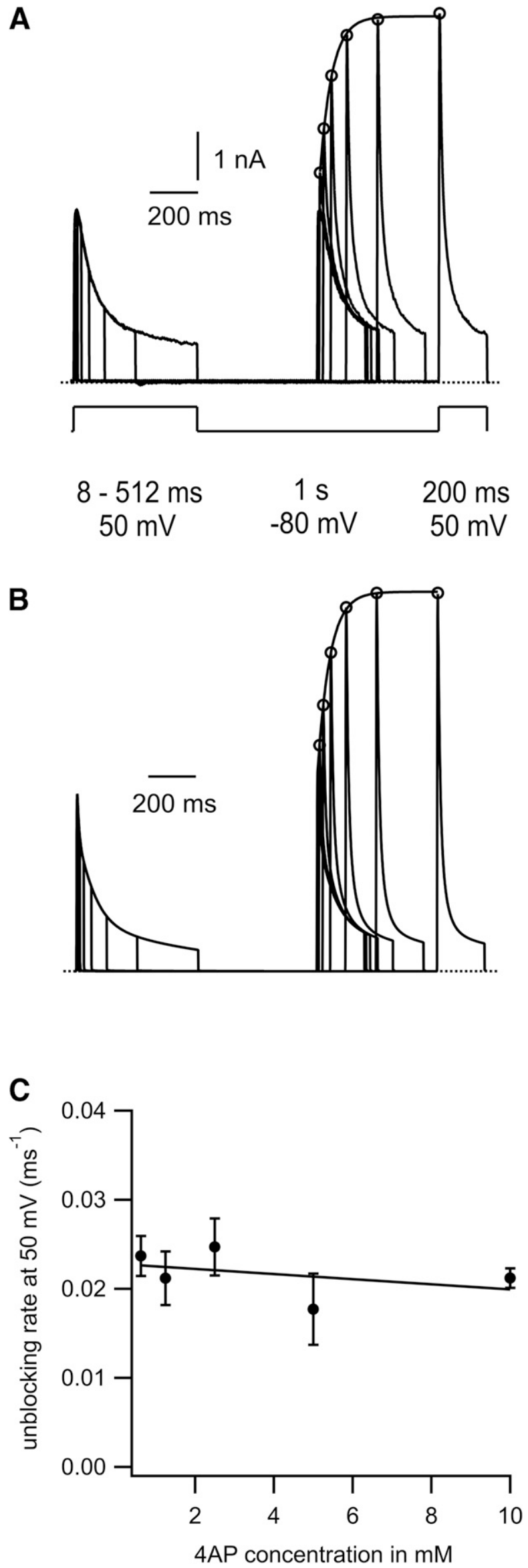

the interval at $-80 \mathrm{mV}$ between the end of the first pulse, and the start of the second or test pulse was 1 second. The duration of the test pulse to $50 \mathrm{mV}$ was 200 milliseconds. For the exemplar traces of Fig. 3A, which were recorded with internal $2.5 \mathrm{mM} 4 \mathrm{AP}$, the envelope of the peak test pulse currents $(\bigcirc)$ was well fitted by a single exponential (solid line) with a time constant, $\tau_{\text {unblock}}$, of 50 milliseconds. In seven cells in which it was measured, $\tau_{\text {unblock }}$ at $50 \mathrm{mV}$ was $41 \pm 4.9$ milliseconds.

Given that the unblocking rate is equal to the sum of the on-rate $\left([4 \mathrm{AP}] * k_{o n}\right)$ and the off-rate $\left(k_{o f f}\right)$, then if $4 \mathrm{AP}$ is unable to bind to open channels at $50 \mathrm{mV}$ then the unblocking rate is expected to be concentration-independent. Figure 3C shows the unblocking rate, calculated as the reciprocal of $\tau_{\text {unblock}}$, as a function of the 4AP concentrations ranging from 0.625 to $10 \mathrm{mM}$. A fit of the data points to a line gave a slope of $-0.0002 \pm 0.0004 \mathrm{mM}^{-1} \mathrm{~ms}^{-1}$, which was not significantly different from zero. This finding that the slope of the fitted line is concentration-independent suggests that little or no binding of $4 \mathrm{AP}$ occurs at $50 \mathrm{mV}$ and that the unblocking rate is determined by $k_{\text {off }}$. This implies that with a 256 millisecond depolarization to $50 \mathrm{mV}$ more than $99 \%$ of channels liganded prior to the conditioning pulse will become unliganded.

4AP Binding at $\mathbf{- 8 0} \mathbf{~ m V}$. Determination of the time and concentration dependence of $4 \mathrm{AP}$ binding at $-80 \mathrm{mV}$ involved a two-pulse protocol in which the duration and the voltage of both pulses were the same ( 300 milliseconds and $50 \mathrm{mV})$, and the interpulse interval of 50 millisecond for the first sweep was increased 1.5-fold on successive sweeps. The rationale for the protocol is that a 300 -millisecond prepulse to $50 \mathrm{mV}$ causes unbinding of $4 \mathrm{AP}$ and ultimately drives most of the channels to the closed inactivated state (Bähring et al., 2001). Returning to $-80 \mathrm{mV}$ between pulses enables inactivated channels to recover to closed states and thence become targets for $4 \mathrm{AP}$ binding. Figure 4A shows representative traces recorded with internal $2.5 \mathrm{mM}$ 4AP. The envelope of the peak test pulse currents shows a biphasic pattern that was well fitted by a rising $\left(\tau_{1}\right)$ and a decaying $\left(\tau_{2}\right)$ exponential function (eq. 4). For the traces in Fig. $4 \mathrm{~A}$, the value for $\tau_{1}$ was $259 \pm 15$ milliseconds, which is a rough approximation of the time constant for recovery from inactivation, and $\tau_{2}$, representing the time constant of $4 \mathrm{AP}$ binding at $-80 \mathrm{mV}$, was $3.3 \pm 0.3$ second. Figure $4 \mathrm{~B}$ shows the output of scheme $4 \mathrm{AP}$ using the same voltage protocol and the same $4 \mathrm{AP}$ concentration as for the experimental data in Fig. 4A.

Fig. 3. The time dependence of the dissociation of $4 \mathrm{AP}$ from $\mathrm{Kv} 4.2$ channels during a depolarization to $50 \mathrm{mV}$. (A) For the experimental traces, a prepulse to $50 \mathrm{mV}$ with a duration that varied from 8 to 512 milliseconds was followed, after a fixed 1 second interval at $-80 \mathrm{mV}$, by a step back to $50 \mathrm{mV}$ for 200 milliseconds. The 1-second interpulse interval at $-80 \mathrm{mV}$ is sufficient to allow near complete recovery from inactivation but is too short to allow substantial rebinding of $4 \mathrm{AP}$, which was present at a concentration of $2.5 \mathrm{mM}$ in the internal solution. The envelope of peak amplitudes of test currents is well fitted by a single exponential with a time constant of 50 milliseconds $($ S.D. $=2.4$ milliseconds). As indicated by the overlap of the prepulse currents, a 40-second interval between sweeps allowed steady-state block to be re-established. (B) For traces simulated using Scheme 4AP (Fig. 7), the voltage protocol and $4 \mathrm{AP}$ concentration were the same as for (A). (C) A plot of the experimental unblocking rate $\left(\mathrm{ms}^{-1}\right)$ at $50 \mathrm{mV}$ versus the concentration of $4 \mathrm{AP}$, which varied from 0.625 to $10 \mathrm{mM}$. The slope of the line fitted to the data does not differ from zero and signifies that the unblocking rate is determined by the dissociation rate, implying that open Kv4.2 channels have little or no affinity for 4AP. 

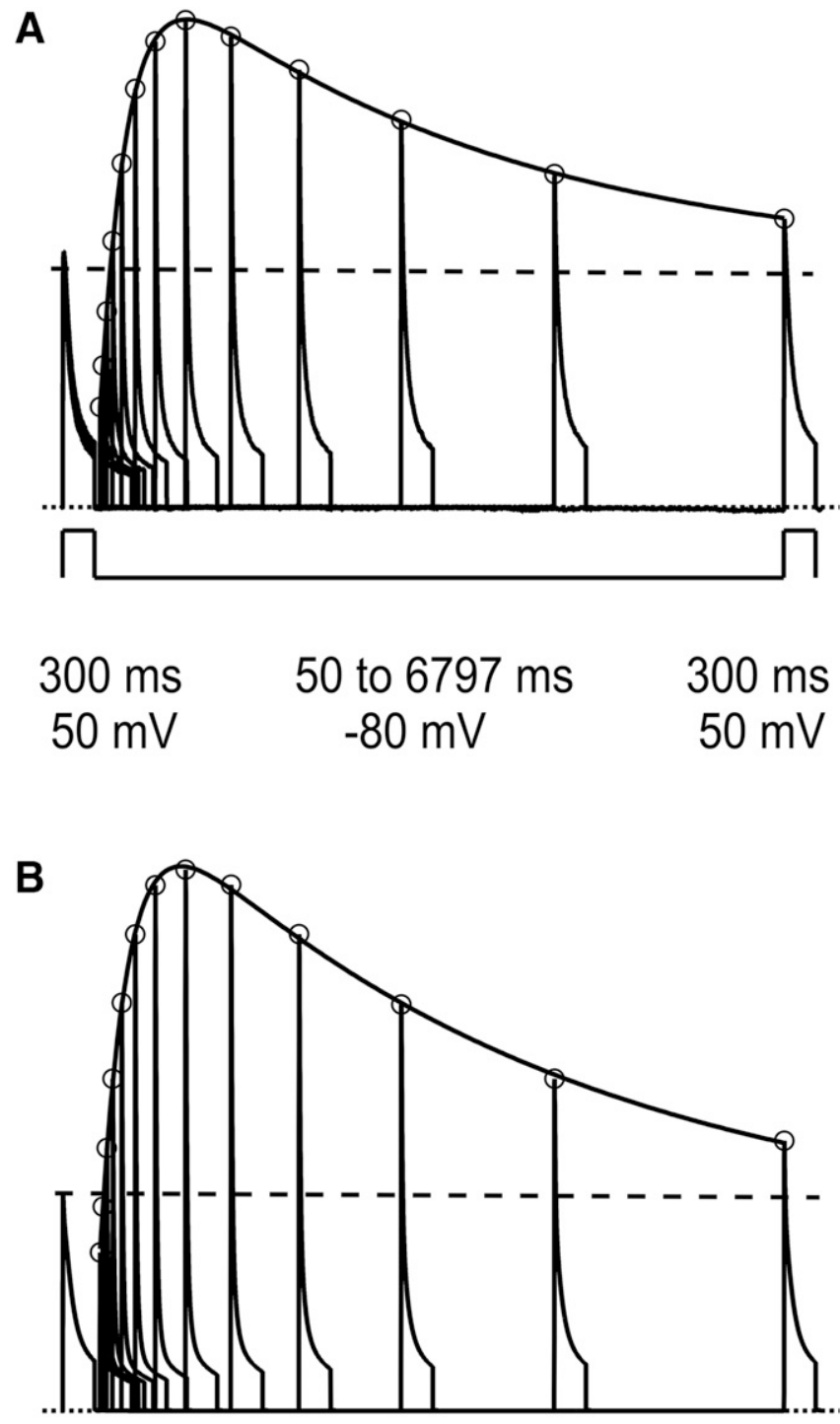

C

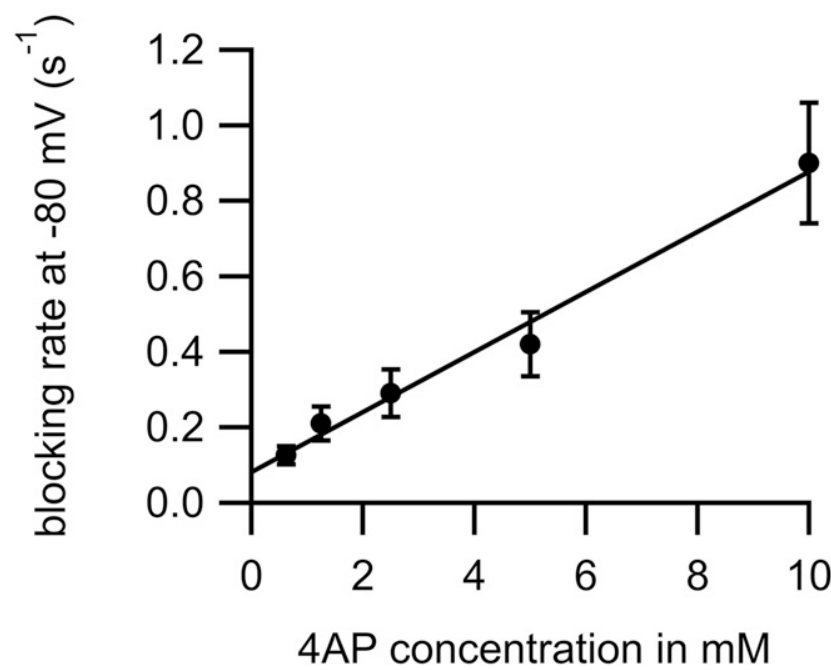

Fig. 4. The time and concentration dependence of the $4 \mathrm{AP}$ binding at $-80 \mathrm{mV}$. (A) The first or conditioning pulse of a two-pulse protocol shown below the current traces triggered the unbinding of $4 \mathrm{AP}$ (cf. Fig. 3A),
For the graph of Fig. 4C the blocking rate, calculated as the reciprocal of the time constant for $4 \mathrm{AP}$ binding ( $\tau_{2}$ of eq. 4 ), is plotted against the concentration of $4 \mathrm{AP}$. Because the blocking rate is equal to the sum of the on-rate and the off-rate at $-80 \mathrm{mV}$, and since only the on-rate has concentration dependence, the $y$-intercept value of $0.08 \pm 0.03$ second $^{-1}$ estimates the value for $k_{\text {off }}$. Consistent with other reports (Castle and Slawsky, 1993; Tseng et al., 1996), the $k_{\text {off }}$ of 0.00008 millisecond $^{-1}(=0.08$ second $\left.^{-1}\right)$ at $-80 \mathrm{mV}$ is much smaller than at $50 \mathrm{mV}(0.021 \pm$ 0.001 millisecond $^{-1}$, Fig. 3C). The slope of the fitted line in Fig. $4 \mathrm{C}$ gives a value of $0.08 \pm 0.005 \mathrm{mM}^{-1} \mathrm{~s}^{-1}$ for $k_{\text {on }}$.

Use-Dependent Unblock. Having established that the unbinding of 4AP from Kv4.2 channels is rapid and probably complete during a 300-millisecond step to $50 \mathrm{mV}$ (Fig. 3), that $k_{\text {on }}$ at $-80 \mathrm{mV}$ is small, and that binding proceeds only after recovery from inactivation (Fig. 4), a more tractable explanation for the increased amplitude of test currents evoked by P2-P4 in the traces of Fig. 5A is possible. The latter phenomenon is representative of reverse use-dependent block or, equivalently, use-dependent unblock, which denote that, within limits, an increase of stimulus frequency causes an increase in the peak current. This use-dependent increase in current arises for the following reasons. For the P1 current of Fig. 5A, which was recorded after a 40 seconds' sojourn at $-80 \mathrm{mV}$ in the presence of $20 \mathrm{mM} 4 \mathrm{AP}$, most of the channels are blocked at the start of the pulse to $50 \mathrm{mV}$ (as suggested by the absence of a fast-activating component of current), but the strong depolarization induces channels first to unblock and then to inactivate. When the membrane potential is returned to $-80 \mathrm{mV}$, channels recover from inactivation and binding of $4 \mathrm{AP}$ to closed channels proceeds, albeit slowly, and, because a depolarizing pulse is applied before the binding reaction achieves steady state, more channels are in closed-unbound states, and the test current is larger.

Since the binding rate is proportional to the $4 \mathrm{AP}$ concentration [i.e., [4AP] $\times k_{o n}(-80 \mathrm{mV})$ ], the extent of $4 \mathrm{AP}$ binding at the end of the 950-millisecond interval between pulses is governed by the 4AP concentration. This is confirmed in the graph in Fig. 5C, which plots the concentration dependence of the mean normalized experimental P2-P4 amplitudes (O) evoked using the voltage protocol described for Fig. 5A. These data indicate that increasing the stimulus frequency to $0.8 \mathrm{~Hz}$ causes an apparent increase of the $K_{d}$ for the block such that $1 \mathrm{mM} 4 \mathrm{AP}$ caused only $8 \%$ inhibition as opposed to $\sim 40 \%$ inhibition for tonic block (Fig. 2), and with $20 \mathrm{mM} 4 \mathrm{AP}$, this changed from $\sim 70 \%$ inhibition for tonic block to $\sim 42 \%$ inhibition at $0.8 \mathrm{~Hz}$. As will be discussed later, the experimental data obtained using the voltage protocol of Fig. 5A are reasonably replicated schematically $4 \mathrm{AP}$ (Fig. $5 \mathrm{~B}$ and $\nabla$ in Fig. 5C).

which then allowed inactivation to proceed. Using eq. 4, a fit of the envelope of the peak test currents $(O)$, evoked by stepping to $50 \mathrm{mV}$, indicated a time constant $\left(\tau_{1}\right)$ for recovery from inactivation of 259 milliseconds and, with $2.5 \mathrm{mM} 4 \mathrm{AP}$ in the internal solution, a time constant $\left(\tau_{2}\right)$ of 3.3 seconds for the rebinding of $4 \mathrm{AP}$ to closed channels at $-80 \mathrm{mV}$. The dashed line denotes the mean of the peak currents evoked by the conditioning pulse. (B) Simulated currents using $2.5 \mathrm{mM} 4 \mathrm{AP}$ and the same voltage protocol gave values of 259 milliseconds and 3.9 seconds, respectively, for $\tau_{1}$ and $\tau_{2}$. See the last section of Results for details. (C) Data obtained using the same experimental protocol as in (A) with concentrations of $4 \mathrm{AP}$ ranging from 0.625 to $10 \mathrm{mM}$. The slope of the line fitted to the data indicates a value for $k_{\text {on }}$ of $0.08 \mathrm{mM}^{-1} \mathrm{~s}^{-1}$, and the $y$-intercept indicates a value for $k_{\text {off }}$ of 0.08 second $^{-1}$. 


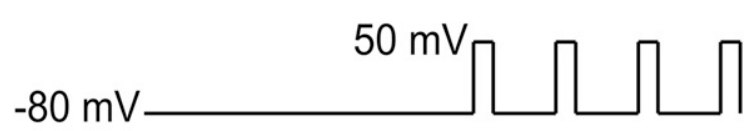

40-60s P1 P2 P3 P4

A

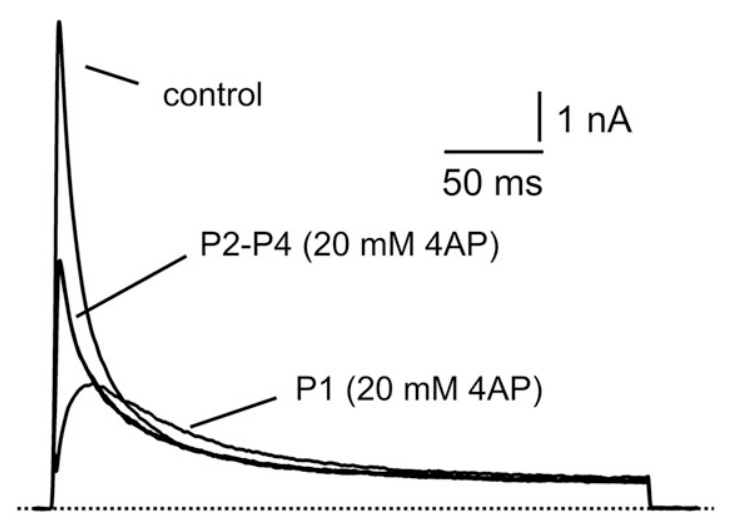

B
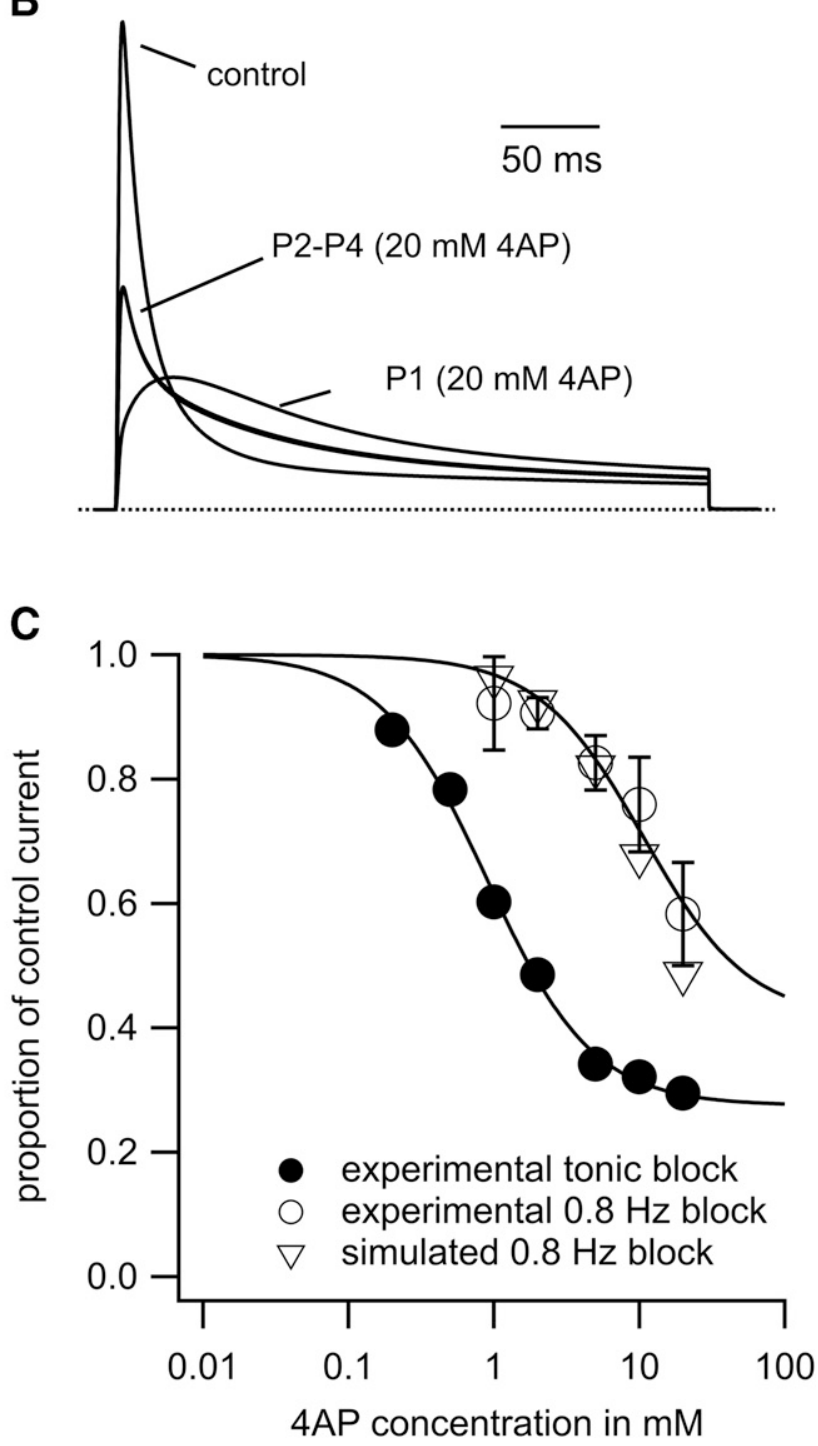

Fig. 5. Increasing the frequency of depolarizing pulses decreases $4 \mathrm{AP}$ block. (A) The voltage protocol shown above the current traces consisted of
Voltage Dependence of the Relative Block of Kv4.2. The aim of this section is to describe the effect of varying the membrane potential in the range of -80 to $-30 \mathrm{mV}$ on test current amplitude in the presence of different concentrations of $4 \mathrm{AP}$. The rationale for the voltage protocol (see Fig. 6B), which is predicated by the mutual exclusivity of inactivation and 4AP block (Fig. 4; see Introduction), was to apply a 30-second depolarizing conditioning step (P1) to increase the extent of channel inactivation and, in doing so, decrease the proportion of closed-bound channels. At the end of the conditioning pulse, the voltage was stepped to $-80 \mathrm{mV}$ for 1.3 seconds to allow the inactivated channels to recover to closed states before the 300 -millisecond test pulse to $50 \mathrm{mV}$. Representative responses in the presence of $10 \mathrm{mM} 4 \mathrm{AP}$ (Fig. 6B) show that the currents after conditioning pulses of -80 and $-70 \mathrm{mV}$ are superimposed, that a step to $-60 \mathrm{mV}$ slightly increases the rise time of the current but has no substantial effect on the peak current, and that the maximal test current is seen after conditioning pulses to either -40 or $-30 \mathrm{mV}$. Test currents such as these were normalized according to eq. 5 to give the relative block, which was then plotted against the potential of the conditioning pulse (P1). Figure $6 \mathrm{~A}$ shows the best fit of a Boltzmann function (eq. 6) to the relative block with $2 \mathrm{mM} 4 \mathrm{AP}\left(V_{\text {half }}=-56.4 \pm 0.4 \mathrm{mV}, s=4.2 \pm 0.3 \mathrm{mV}, n=6\right)$ and $10 \mathrm{mM} 4 \mathrm{AP}\left(V_{\text {half }}=-51.8 \pm 0.2 \mathrm{mV}, s=3.3 \pm 0.4 \mathrm{mV}, n=10\right)$. As noted by Thompson (1982) and others, the relative block curves have a shape similar to that for the availability curve (dashed line of Fig. 6A) but show a concentration-dependent rightward shift of the $V_{\text {half }}$, the voltage at which the relative block is half-maximal (see Table 1 for the experimental and simulated values for $V_{\text {half }}$ and $s$ ). Figure $6, \mathrm{C}$ and D, show the outcome of the relative block voltage protocol in the gating model and are discussed along with other simulated data in the following section.

A Model of 4AP Block of Kv4.2. The effect of 4AP on Kv4.2 currents has not been modeled previously; however, several gating models have been proposed to account for the block by $4 \mathrm{AP}$ of transient outward currents, such as $I_{t o}$ and $I_{A}$. The first, a five-state model incorporating both closed and open channel block (Thompson, 1982) and a related four-state model engendering only closed-state block (Kehl, 1990), reproduced several of the properties of $4 \mathrm{AP}$ block but were predicated on the assumption, now known to be incorrect, that a single inactivation process was strictly coupled to channel

a 40 - to 60 -second period at $-80 \mathrm{mV}$, followed by four 300 -millisecond pulses to $50 \mathrm{mV}$ separated by a 950-millisecond interpulse interval at $-80 \mathrm{mV}$. The peak amplitude of the first pulse (P1), taken to represent tonic block, was used to generate the concentration-response relationship of Fig. 2. Current traces recorded in the continuous presence of external $20 \mathrm{mM} 4 \mathrm{AP}$ show a $56 \%$ increase in the peak current of pulses $2-4$ relative to pulse 1 , as well as a marked increase of the activation rate. (B) The output in Scheme 4AP using the same voltage protocol and $20 \mathrm{mM} 4 \mathrm{AP}$ reproduces the experimental data reasonably well. (C) The mean ( \pm S.E.) of the peak currents evoked by P2-P4 expressed as a proportion of the control current vs. the $4 \mathrm{AP}$ concentration. There is reasonable concordance of the experimental $(O)$ and simulated $(\nabla)$ relationship except with the higher concentrations of $4 \mathrm{AP}$, where the simulated currents tend to show slightly less potentiation than the experimental currents. Comparison with the data for the tonic block $(\bullet)$, taken from Fig. 2 , indicates that increasing the stimulus frequency causes an apparent right shift of the concentration-response curve. The Boltzmann function fitted to the data for the experimental $0.8 \mathrm{~Hz}$ block has a $V_{\text {half }}$ of $10.7 \pm 0.45 \mathrm{mM}$ and a base of $0.41 \pm 0.1$ with $h$ fixed at 1.2 

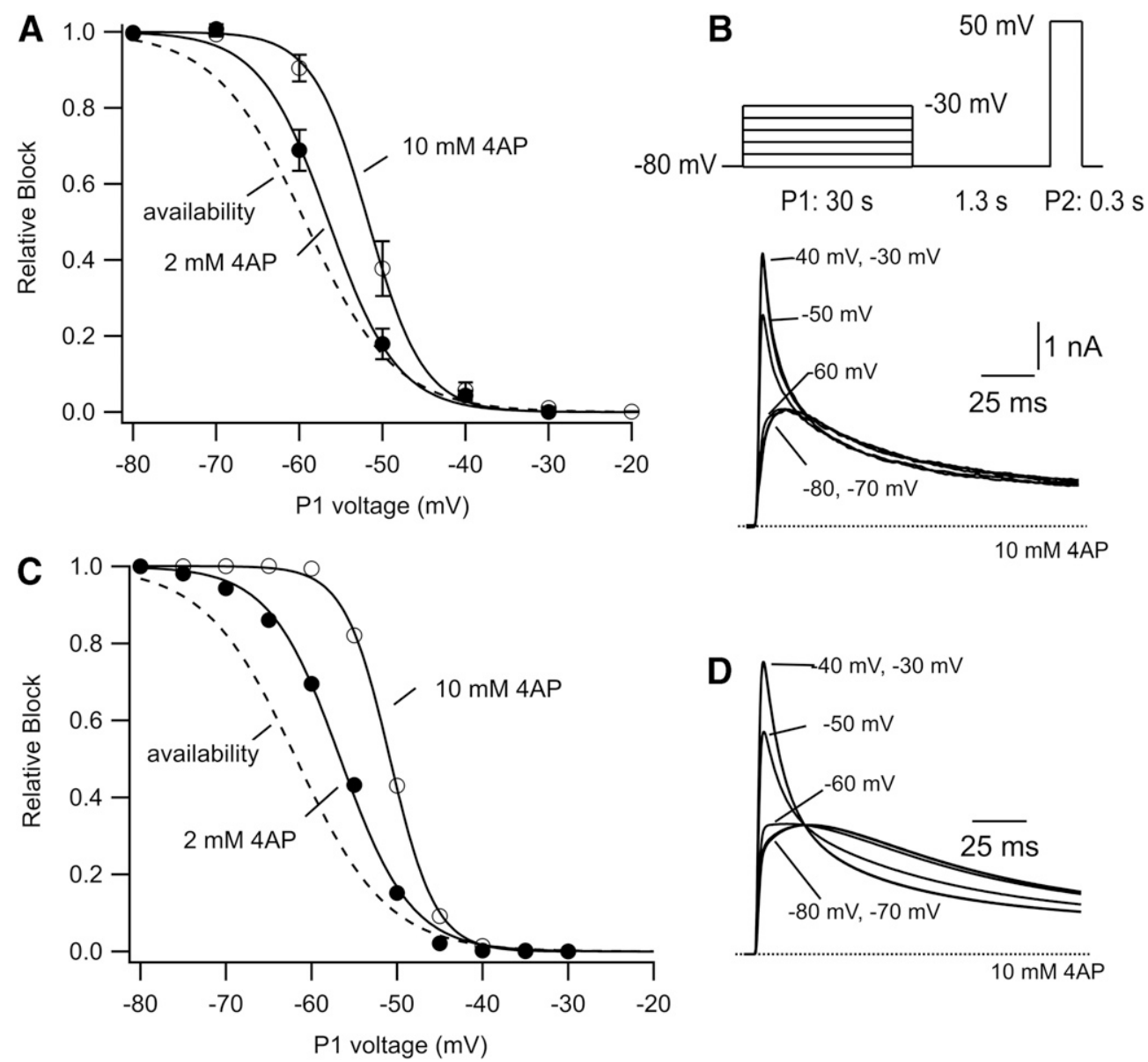

Fig. 6. Changing the holding potential between -80 and $-30 \mathrm{mV}$ causes a concentration-dependent decrease in the $4 \mathrm{AP}$ block. (A) The experimental relative block, calculated according to eq. 5, as a function of the P1 (conditioning) voltage of the stimulus protocol, which is shown uppermost in (B). With respect to the control availability curve, the $V_{\text {half }}$ derived from a fit of the data to a Boltzmann function (eq. 6 ) shifted by 3.1 and $7.5 \mathrm{mV}$ in 2 and $10 \mathrm{mM}$ $4 \mathrm{AP}$, respectively. The shift of the $V_{\text {half }}$ was associated with a decrease of the slope factor from the mean control value of 5.4-4.2 and 3.3 $\mathrm{mV}$ in 2 and $10 \mathrm{mM} 4 \mathrm{AP}$, respectively. (B) Representative experimental P2 currents evoked in $10 \mathrm{mM} 4 \mathrm{AP}$ at the indicated P1 voltages. (C) The relative block simulated using Scheme 4AP and using the same voltage protocol and 4AP concentrations used to generate the data of (A). Although the $V_{\text {half }}$ for the simulated availability curve is slightly left-shifted with respect to the experimental availability curve (see Table 1 ), the right shift of $V_{\text {half }}$ by $5 \mathrm{mV}$ in $2 \mathrm{mM} 4 \mathrm{AP}$ and by $10.8 \mathrm{mV}$ in $10 \mathrm{mM} 4 \mathrm{AP}$ compares reasonably well to that observed experimentally. With $2 \mathrm{mM} 4 \mathrm{AP}$, the decrease of $s$ by $1.2 \mathrm{mV}$ was the same for the experimental and the simulated data, and with $10 \mathrm{mM} 4 \mathrm{AP}$, the decrease of $s$ in the simulation was $2.6 \mathrm{mV}$ versus $2.1 \mathrm{mV}$ for the experimental data (cf. Table 1). (D) Simulated current traces generated using the same voltage protocol and 4AP concentration as in (B) and from which the relative block curve in $10 \mathrm{mM} 4 \mathrm{AP}$ in $(\mathrm{C})$ was derived.

opening. A subsequent 10-state model (Campbell et al., 1993) reproduced many of the features of block of $I_{t o}$ in ventricular myocytes and incorporated closed-state binding of $4 \mathrm{AP}$, as well as closed-state inactivation, but here, too, some of the assumptions underpinning the gating scheme are now known to be incorrect. For example, the model assumed that closedstate inactivation was in series with open-channel inactivation and, by extension, that the two were conformationally related, but it has been shown that closed-state inactivation (CSI) persists after fast open-state inactivation has been removed (Jerng and Covarrubias, 1997).

Figure 7 shows the gating scheme, referred to subsequently as Scheme $4 A P$, used to simulate the effect of $4 \mathrm{AP}$ on $\mathrm{Kv} 4.2$ currents. The middle and lower rows of the gating scheme are the same as for model 2 of Bähring et al. (2001) that was shown to replicate the biophysical properties of Kv4.2 ionic currents, such as closed-state inactivation, recovery from CSI that bypasses the open state, and three components of inactivation during a 2.5-second depolarization to $40 \mathrm{mV}$. In this study, a third component of inactivation was not detectable with a 300 -millisecond step to $50 \mathrm{mV}$, but there was good concordance between the mean values for and the relative amplitudes of $\tau_{f}$ (7.8 milliseconds and 0.75) and $\tau_{s}$ (40.2 milliseconds and 0.25) and those reported by Bähring et al. (2001) for $\tau_{1}$ (11.0 milliseconds and 0.73) and $\tau_{2}$ (50 milliseconds and 0.23). An allosteric factor $f$, which is required to satisfy microscopic reversibility, and the rate constants were unchanged with one exception: the value for $k_{i c}$ was decreased from 0.1 to $0.02 \mathrm{~s}^{-1}$ to give a time constant for recovery from inactivation at $-80 \mathrm{mV}$ of $330 \pm 8$ milliseconds, which is a good approximation of the experimentally measured value of $330 \pm 14$ milliseconds (not shown). With $k_{i c}=0.02 \mathrm{~second}^{-1}$ the midpoint of the availability or steady-state inactivation curve was $-61.7 \pm 0.1 \mathrm{mV}$, which is left-shifted by roughly $4 \mathrm{mV}$ with respect to the mean experimental value. Simulated and experimental availability curves had slope factors, determined by the fit to the Boltzmann function (eq. 6), of $5.3 \pm$ 0.2 and $5.4 \pm 0.2$ milliseconds, respectively (Table 1 ). 
TABLE 1

Comparison of the experimental and simulated relative block values (mean \pm S.D.) for the midpoint potential $\left(V_{1 / 2}\right)$ and the slope factor $(s)$ estimated by a least squares fit to a Boltzmann relationship (eq. 6)

\begin{tabular}{lccccc}
\hline & \multicolumn{2}{c}{$V_{1 / 2}$} & & \multicolumn{2}{c}{$s$} \\
\cline { 2 - 3 } \cline { 5 - 6 } & Experimental & Simulated & & Experimental & Simulated \\
\hline Availability & $(\mathrm{mV})$ & $(\mathrm{mV})$ & & $(\mathrm{mV})$ & $(\mathrm{mV})$ \\
Relative block, 2 mM 4AP & $-59.3 \pm 0.3(n=9)$ & $-61.6 \pm 0.1$ & & $5.4 \pm 0.2(n=9)$ & $5.3 \pm 0.2$ \\
Relative block, 10 mM 4AP & $-51.8 \pm 0.4(n=6)$ & $-56.6 \pm 0.2$ & & $4.2 \pm 0.3(n=6)$ & $4.1 \pm 0.2$ \\
\cline { 1 - 3 }
\end{tabular}

The middle row of Scheme 4AP includes an activation pathway that presumes a tetrameric channel construct comprising four identical but independent subunits, a concerted opening transition from the fully activated not-open or preopen closed state $\left(\mathrm{C}_{4}\right)$, and open channel inactivation arising from $\mathrm{N}$-type inactivation in series with $\mathrm{C}$-type inactivation. Downward and upward transitions between the middle and lower row of states represent CSI and recovery from CSI, respectively. Transitions between states in the lower row represent activation and deactivation of closedinactivated channels.

Block by $4 \mathrm{AP}$ was incorporated by the addition of six $4 \mathrm{AP}$ bound states $\left(\mathrm{C}_{0} \mathrm{~B}\right.$ to $\mathrm{OB}$, top row of Fig. 7). Binding of $4 \mathrm{AP}$ is restricted to closed states $\left(\mathrm{C}_{0} \mathrm{~B}\right.$ to $\left.\mathrm{C}_{4} \mathrm{~B}\right)$, and the on-rate is the product of the association rate constant $\left(k_{o n}\right)$, in $\mathrm{mM}^{-1} \mathrm{~s}^{-1}$, and the 4AP concentration (in $\mathrm{mM}$ ). This assumption of a $1: 1$ binding stoichiometry is consistent both with the concentration-response relationship reported here (Fig. 2) and by others for Kv4-based rapidly inactivating currents (Castle and Slawsky, 1993; Tseng et al., 1996), as well as for the block of other classes of Kv channels (e.g., Kirsch and Drewe, 1993). Based on the data in Fig. 3C, the open state is presumed to have no affinity for 4AP. In the Shaker channel, the movement of the cationic form of $4 \mathrm{AP}$ onto its binding site is affected by the membrane electric field ( $e$-fold change per $58 \mathrm{mV}$ at $20^{\circ} \mathrm{C}$ ); however, the binding reaction is assumed here not to be directly voltage-dependent. To satisfy microscopic reversibility, it was also necessary to include an allosteric factor, $g$, both in the vertical transition rates representing $4 \mathrm{AP}$ binding and unbinding between the middle and top rows of Scheme 4AP and in the horizontal transitions representing voltage sensor activation between closed-bound states. Several assumptions were made to decrease the number of free variables in the model. The first is that voltage sensor movement is unchanged in 4AP-bound closed channels. No data are available on the effect of $4 \mathrm{AP}$ on $\mathrm{Kv} 4.2$ gating currents, but this assumption is consistent with numerical modeling showing that the features of the 4AP block of the delayed rectifier of the SGA can be replicated without the need to change activation gating kinetics (Yeh et al., 1976a) and

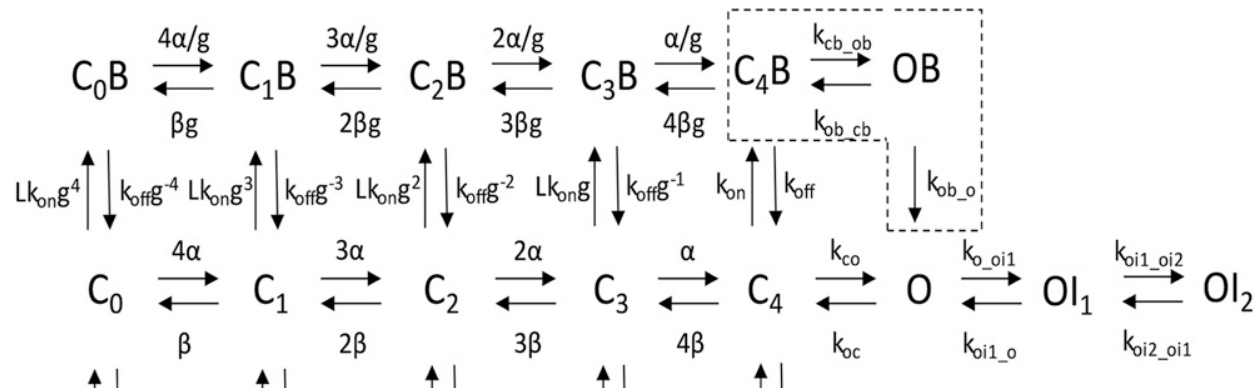

Scheme 4AP

$$
\mathrm{Cl}_{0} \underset{\beta \mathrm{f}}{\stackrel{4 \alpha / \mathrm{f}}{\rightleftarrows}} \mathrm{Cl}_{1} \underset{2 \beta \mathrm{f}}{\stackrel{3 \alpha / \mathrm{f}}{\rightleftarrows}} \mathrm{Cl}_{2} \underset{3 \beta \mathrm{f}}{\stackrel{2 \alpha / \mathrm{f}}{\rightleftarrows}} \mathrm{Cl}_{3} \underset{4 \beta \mathrm{f}}{\stackrel{\alpha / \mathrm{f}}{\rightleftarrows}} \mathrm{Cl}_{4}
$$

Fig. 7. A model, referred to as Scheme $4 \mathrm{AP}$, of the block of Kv4.2 by $4 \mathrm{AP}$. The model was built around the gating scheme referred to as model 2 in Bähring et al. (2001), which comprises the middle and lower rows of channel states. Activation of closed channels (middle row) involves voltage sensor movement in each of the subunits of the tetrameric structure and leads to the activated-not-open or preopened closed state $\left(\mathrm{C}_{4}\right)$. Channel opening and channel closing result from concerted (highly cooperative) transitions. In the same row, two forms of voltage-independent inactivation, presumed to involve the $\mathrm{N}$ terminus $\left(\mathrm{OI}_{1}\right)$ and the outer pore region $\left(\mathrm{OI}_{2}\right)$, are coupled to channel opening. Closed-state inactivation (CSI), is represented by the voltage-independent vertical transitions between the middle and lower row; voltage sensor activation is facilitated in inactivated channels $(f=0.3)$. During a strong depolarization, channels ultimately accumulate in $\mathrm{CI}_{4}$ in a process that has been referred to as preferential CSI. The modification to model 2 of Bahring et al. (2001) is the addition of binding/unbinding of 4AP, represented by the vertical transitions between the upper and middle rows. Note that in this reaction scheme, 4AP binding and CSI are explicitly mutually exclusive processes: inactivated channels do not bind 4AP, and 4AP-bound channels do not inactivate. Setting $g$ to 1 causes voltage sensor movement to be the same in the top and middle rows, and it makes the affinity of $4 \mathrm{AP}$ for the closed states the same. The presumption that $4 \mathrm{AP}$ has no affinity for the OB state is based on the experimental observation that the unblocking rate during a step to $50 \mathrm{mV}$ (see Fig. 3C) has no concentration dependence. See Table 2 for the details of the simulation parameters. As discussed in the text, two approaches were taken with respect to the gating transitions bounded by the dashed lines. The outcome of the simulation was the same whether the $\mathrm{OB}$ to $\mathrm{O}$ transition was assumed to be rate-limiting or if stabilization of the preopen closed state $\left(\mathrm{C}_{4} \mathrm{~B}\right)$ and destabilization of the $\mathrm{OB}$ state caused channel opening to be the rate-limiting step. 
that SGA delayed rectifier gating currents (Spires and Begenisich, 1989), as well as Shaker gating currents (McCormack et al., 1994; Armstrong and Loboda, 2001), are unaffected by 4AP. To satisfy this presumption that the activation kinetics of closed-bound channels are the same as for closed-unbound channels, it was necessary to constrain the allosteric factor $g$ to be 1 . Because $g$ also appears in the calculation of the on and off rates for 4AP binding, another consequence of setting $g$ to 1 is that the affinity of $4 \mathrm{AP}$ for each of the five closed states $\left(\mathrm{C}_{0}-\mathrm{C}_{4}\right)$ is the same. Some support for this assumption has been provided by Campbell et al. (1993), who found that the affinities were not substantially different for at least two of the closed states. Constraining $g$ to be 1 means that it could be eliminated from the gating scheme, but it is nonetheless included to give an appreciation of the fact that a value for $g$ greater than or less than 1 affects both the activation kinetics of closed-bound states and the relative affinities of the closed states for 4AP.

In all the previous models of the $4 \mathrm{AP}$ block of transient outward currents (Thompson, 1982; Kehl, 1990; Campbell et al., 1993), 4AP dissociation was assumed to precede channel opening. When this same condition was imposed in Scheme 4AP by eliminating the open blocked state, there was a concentration-dependent decrease in the peak current evoked by a step from -80 to $50 \mathrm{mV}$; however, the characteristic apparent slowing of activation and inactivation that culminate in the crossover of control and treated currents did not occur, regardless of the value for $g$ (not shown). Consequently, to replicate the effects of $4 \mathrm{AP}$ on $\mathrm{Kv} 4.2$ current kinetics, it was necessary to include transitions from the closed-blocked to the open-blocked to the open state (corresponding to $\mathrm{C}_{4} \mathrm{~B}$ to $\mathrm{OB}$ to $\mathrm{O}$ in Fig. 7). In the case of delayed rectifier-like $\mathrm{K}^{+}$channels, two different approaches have been used to model the time course of these transitions. For the $4 \mathrm{AP}$ block of the SGA delayed rectifier, unbinding of $4 \mathrm{AP}$ from the open state was assumed to be the rate-limiting step (Yeh et al., 1976a), whereas for the block of inactivationremoved Shaker channels the opening step was assumed to be rate-limiting (Armstrong and Loboda, 2001). With Scheme 4AP, either of these approaches, normal opening and slow unblock or slow opening and rapid unblock, produced identical outcomes (not shown); and, for reasons that are discussed later, the choice was made to set the unblocking of open-bound channels as the rate-limiting step (Table 2).

Since, with the one exception noted above with respect to $k_{i c}$, the transition rates for the middle and bottom row of states of Scheme 4AP were constrained to be the same as those reported by Bähring et al. (2001), and in light of the constraint that the allosteric factor $g$ be fixed at 1 , the addition of six 4APbound states used to generate Scheme 4AP added five variables $\left(k_{o n}, k_{o f f}, k_{c b \_o b}, k_{o b \_c b}\right.$, and $\left.k_{o b \_o}\right)$, of which two, $k_{o n}$ and $k_{\text {off }}$ at $-80 \mathrm{mV}$, were constrained by experimental data. Table 2 lists the values of the simulation variables.

Overall, Scheme 4AP reproduces the time and concentration dependence of the effect of $4 \mathrm{AP}$ on $\mathrm{Kv} 4.2$ currents reasonably well. The simulated values for the concentration-response relationship (dashed line in Fig. 2) yield a $K_{d}$ of $0.88 \pm 0.06 \mathrm{mM}$, a Hill coefficient $(h)$ of $1.16 \pm 0.07$, and a base of $0.25 \pm 0.02$, which represent the proportion of the normalized peak current persisting in a saturating concentration of $4 \mathrm{AP}$. These correspond well with the experimental values in Fig. $2(0.9 \pm 0.07 \mathrm{mM}, 1.2 \pm$ 0.07 , and $0.28 \pm 0.02$, respectively) and are in accord with those
TABLE 2

Parameter values for Scheme 4AP in which 4AP dissociation from openbound channels is rate-limiting

\begin{tabular}{|c|c|c|}
\hline Parameter & Value & Source \\
\hline$\alpha$ & $200 \mathrm{~s}^{-1}(0 \mathrm{mV})$ & Bähring et al., 2001 \\
\hline$z_{\alpha}$ & 1.0 & Bähring et al., 2001 \\
\hline$\beta$ & $4 \mathrm{~s}^{-1}(0 \mathrm{mV})$ & Bähring et al., 2001 \\
\hline$z_{\beta}$ & 1.58 & Bähring et al., 2001 \\
\hline$k_{c o}$ & $400 \mathrm{~s}^{-1}$ & Bähring et al., 2001 \\
\hline$k_{o c}$ & $1100 \mathrm{~s}^{-1}$ & Bähring et al., 2001 \\
\hline$k_{o \_o i 1}$ & $300 \mathrm{~s}^{-1}$ & Bähring et al., 2001 \\
\hline$k_{\text {oi1_o }}$ & $30 \mathrm{~s}^{-1}$ & Bähring et al., 2001 \\
\hline$k_{o i 1 \_o i 2}$ & $30 \mathrm{~s}^{-1}$ & Bähring et al., 2001 \\
\hline$k_{\text {oi2_oi1 }}$ & $15 \mathrm{~s}^{-1}$ & Bähring et al., 2001 \\
\hline$k_{c i}$ & $30 \mathrm{~s}^{-1}$ & Bähring et al., 2001 \\
\hline$f$ & 0.3 & Bähring et al., 2001 \\
\hline$k_{i c}$ & $0.02 \mathrm{~s}^{-1}$ & See text \\
\hline$k_{\text {on }}$ & $0.08 \mathrm{mM}^{-1} \mathrm{~s}^{-1}(-80 \mathrm{mV})$ & Fig. $4 \mathrm{C}$ \\
\hline$L$ & {$[4 \mathrm{AP}]$} & \\
\hline$k_{\text {off }}$ & $0.08 \mathrm{~s}^{-1}(-80 \mathrm{mV})$ & Fig. $4 \mathrm{C}$ \\
\hline$g$ & 1 & \\
\hline$k_{c b \_o b}$ & $=k_{c o}$ & \\
\hline$k_{o b \_c b}$ & $=k_{o c}$ & \\
\hline$k_{o b \_o}$ & $100 \mathrm{~s}^{-1}$ & \\
\hline
\end{tabular}

reported for Kv4.2 channels expressed in Xenopus oocytes $\left(K_{d}=1.5 \mathrm{mM}\right.$, base $\left.\approx 0.28\right)$ (Tseng et al., 1996).

The concentration dependence of the decay kinetics is also replicated by the model. For example, in the absence of $4 \mathrm{AP}$, the decay during a 300-millisecond step to $50 \mathrm{mV}$ was well fitted by a biexponential function with time constants of 10.3 and 47.3 millisecond (vs. experimental values of $7.8 \pm 0.2$ and $40.2 \pm 1.4$ milliseconds), and in $10 \mathrm{mM} 4 \mathrm{AP}$, the decay of the modeled current was well fitted by a monoexponential function with a time constant of 65.6 milliseconds (vs. the experimental value of $58.1 \pm 1.9$ milliseconds, $n=10$ ). This change in the decay rate with high concentrations of $4 \mathrm{AP}$ appears to arise primarily from the much slower delivery of channels to the N-type inactivation process $\left(\mathrm{OI}_{1}\right.$ in Scheme 7$)$. A similar explanation has been proposed to account for a comparable action of 4AP to slow C-type inactivation in ShIR channels (Castle et al., 1994b).

Using a voltage protocol identical to that shown in Fig. 3A, the model accurately replicates the time dependence of the channel unblock at $50 \mathrm{mV}$ (Fig. $3 \mathrm{~B}, \tau_{\text {exp }}=50 \pm 2.4$ milliseconds vs. $\tau_{\text {sim }}=51.6 \pm 1.2$ milliseconds), as well as an $\sim 2.1$-fold increase in the test pulse current, relative to the peak of the prepulse current, after the 256- and 512-millisecond prepulses. Likewise, in Fig. 4, A and B, agreement was good between the time dependence of recovery from inactivation (which is underestimated by the fitting process) $\left(\tau_{\exp }=\right.$ $259 \pm 15$ milliseconds vs. $\tau_{\text {sim }}=259 \pm 8$ milliseconds) and the onset of block $\left(\tau_{\text {exp }}=3.29 \pm 0.27\right.$ seconds vs. $\tau_{\text {sim }}=3.9 \pm 0.37$ seconds). The maximum test current, expressed as a proportion of the prepulse current, was 2.1 and 2.4 for the experimental and simulated data, respectively.

The model also replicates reverse use-dependent block in which the proportion of channels blocked by $4 \mathrm{AP}$ is decreased by increasing the frequency of test depolarizations. Figure 5B shows there is a reasonable correlation, both in terms of peak amplitude and the kinetics of the currents, between simulated traces presented in the same format, using the same voltage protocol, and with the same $4 \mathrm{AP}$ concentration as for the experimental traces in Fig. 5A; and, in Fig. 5C, a plot of the experimentally measured $(O)$ and the simulated $(\nabla)$ relationship between the average peak amplitude of the P2-P4 test 
currents and the concentration of the 4AP shows that the two are similar.

Finally, the graph in Fig. $6 \mathrm{C}$ shows the effect of 2 and $10 \mathrm{mM}$ $4 \mathrm{AP}$ on the relationship between the relative block and the voltage of a 30 -second conditioning prepulse (P1). Data points of Fig. 6C were measured from simulated currents, such as those in Fig. 6D. In Fig. 6C, the dashed line represents the simulated control steady-state availability curve $\left(V_{\text {half }}=\right.$ $-61.6 \pm 0.1 \mathrm{mV}, s=5.3 \pm 0.2 \mathrm{mV}$ ) for the model, and the solid lines represent the best fit of the data to a Boltzmann relationship (eq. 6) in $2 \mathrm{mM} 4 \mathrm{AP}\left(V_{\text {half }}=-56.6 \pm 0.2 \mathrm{mV}\right.$, $s=4.1 \pm 0.2 \mathrm{mV})$ and $10 \mathrm{mM} 4 \mathrm{AP}\left(V_{\text {half }}=-50.8 \pm 0.1 \mathrm{mV}, s=2.7\right.$ $\pm 0.1 \mathrm{mV})$. With respect to the simulated availability curve, this finding indicates that the $V_{\text {half }}$ of the simulated relative block relationship is right-shifted by $\sim 5.1 \mathrm{mV}$ in $2 \mathrm{mM} 4 \mathrm{AP}$ and by $\sim 10.9 \mathrm{mV}$ in $10 \mathrm{mM} 4 \mathrm{AP}$; the simulated curve in $10 \mathrm{mM}$ $4 \mathrm{AP}$ is right-shifted by $5.8 \mathrm{mV}$ with respect to the curve in $2 \mathrm{mM}$ 4AP. Table 1 summarizes the experimental and simulated values for $V_{\text {half }}$, and $s$ and indicates reasonable agreement between the two data sets.

\section{Discussion}

Comparison with a Previous Study of the Block of Kv4.2 by 4AP. The experimental data are in good agreement with those reported for Kv4.2 channels expressed in Xenopus oocytes (Tseng et al., 1996). The concentration-response relationship gave values for the $K_{d}$ of $0.9 \mathrm{mM}$ (Fig. 2) versus $1.5 \mathrm{mM}$, and both indicated a value of $\sim 70 \%$ for the maximal block of the current with a saturating concentration of $4 \mathrm{AP}$; the values for $\tau_{\text {unblock }}$ in the two studies are virtually the same: $41 \pm 4.9$ milliseconds with a conditioning pulse to $50 \mathrm{mV}$ versus $42 \pm 6$ milliseconds with a conditioning pulse to $60 \mathrm{mV}$. It was suggested that $\tau_{\text {unblock }}$ at positive voltages was independent of the $4 \mathrm{AP}$ concentration, and data supporting that notion were obtained in this study (Fig. 3C). With respect to the relative block (Fig. 6), the experimental values (Table 1) for the right shift of the $V_{\text {half }}$ going from 2 to $10 \mathrm{mM}$ were comparable (i.e., $4.4 \mathrm{mV}$ versus $5.1 \mathrm{mV}$, as were the values for $s$ with $4 \mathrm{AP}$ concentrations of $2 \mathrm{mM}(4.1 \mathrm{mV}$ vs. $4.1 \mathrm{mV})$ and $10 \mathrm{mM}(3.3 \mathrm{mV}$ vs. $3.7 \mathrm{mV})$. Although the estimates of the unblocking rates at $-80 \mathrm{mV}\left(0.08 \pm 0.03\right.$ second $^{-1}$ (Fig. 4C) vs. 0.062 second $^{-1}$ ) are similar, there is a substantial difference in the association rate constant of $0.08 \pm 0.005 \mathrm{mM}^{-1} \mathrm{~s}^{-1}$ (Fig. $4 \mathrm{C})$, which is 6.6 -fold larger than that reported by Tseng et al. (1996). Overall, the similarity of the data reported in this study and by Tseng et al. (1996) suggests that the values used to constrain Scheme 4AP are reasonable.

Comparison with the Block of $I_{\text {to }}$ by 4AP. In cardiac myocytes, $I_{t o}$ is mediated by $\mathrm{Kv} 4$ channels coexpressed with accessory subunits such as Kv-channel interacting proteins and dipeptidyl aminopeptidase-like proteins (Birnbaum et al., 2004; Niwa and Nerbonne, 2010). Because a major effect of the accessory subunits is to slow the fast phase of inactivation during a strong depolarizing pulse and to accelerate recovery from inactivation, it is of some interest to compare the effects of $4 \mathrm{AP}$ on $\mathrm{Kv} 4.2$ versus cardiac $I_{t o}$. Qualitatively, the features of the block of $\mathrm{I}_{\mathrm{to}}$ by $4 \mathrm{AP}$ are the same as those noted but with some quantitative differences. These include a higher affinity for $4 \mathrm{AP} K_{d} \sim 0.2 \mathrm{mM}$ (Castle and Slawsky, 1993) versus $1 \mathrm{mM}$ and an association rate constant $\left(k_{o n}\right)$ of $0.2 \mathrm{mM}^{-1} \mathrm{~s}^{-1}$ at $-70 \mathrm{mV}$, which is 2.5 - and 17 -fold larger, respectively, than that measured at $-80 \mathrm{mV}$ in this study and by Tseng et al. (1996). Despite the wide variation in these $k_{o n}$ values, the dissociation rate constants $\left(k_{\text {off }}\right)$ from the same three laboratories are quite comparable $\left(0.09\right.$ second $^{-1}$ at $-70 \mathrm{mV}, 0.08$ second $^{-1}$, and 0.062 second $^{-1}$ at $-80 \mathrm{mV}$ ). In light of the similarity of the $k_{\text {off }}$ values at -70 to $-80 \mathrm{mV}$, it is surprising that at $50 \mathrm{mV}$, the value for $k_{\text {off }}$ in ferret myocytes (Campbell et al., 1993) is roughly 5 -fold smaller for $I_{t o}$ than the value of 25 seconds $^{-1}$ measured in $\mathrm{Kv} 4.2$ channels (Fig. 3C). Despite the fact that $k_{\text {off }}$ at depolarized potentials is markedly smaller in $I_{t o}$, it shares with $\mathrm{Kv} 4.2$ (Fig. 3C) the property of being concentration-independent (Castle and Slawsky, 1993) and provides some additional support for the assumption in the proposed gating model that open $\mathrm{Kv} 4.2$ channels do not bind 4AP.

Comparison with the 4AP Block of Other Kv Channels. Many of the features of the block of Kv 4.2 by $4 \mathrm{AP}$ are similar to those reported for the delayed rectifier current, both in the SGA (Yeh et al., 1976a; Kirsch et al., 1986) and its cell bodies in the giant fiber lobe (Jerng and Gilly, 2002), which is putatively mediated by channels of the Kv1 class (Rosenthal et al., 1996). These features include closedchannel block at a membrane voltage as low as $-100 \mathrm{mV}$ (Jerng and Gilly, 2002), implying that binding can occur in the fully deactivated closed state, and a relief of the block $\left(\tau_{\text {unblock }}=10-20\right.$ milliseconds $)$ during membrane depolarization (i.e., use-dependent unblock). Indeed, aside from the fact that Scheme 4AP included inactivation gating, it is qualitatively quite similar to that used by Yeh et al. (1976a). This similarity of these two gating models includes the assumption that activation gating is unaffected by $4 \mathrm{AP}$, as has been shown directly by gating current measurements in the SGA (Spires and Begenisich, 1989) and Shaker (McCormack et al., 1994; Loboda and Armstrong, 2001).

The $k_{o n}$ for 4AP in open Kv3.1 channels is $2.1 \times 10^{4} \mathrm{M}^{-1} \mathrm{~s}^{-1}$ (Kirsch and Drewe, 1993), and that for the open Shaker channel at $0 \mathrm{mV}$ is $1.4 \times 10^{7} \mathrm{M}^{-1} \mathrm{~s}^{-1}$ (Armstrong and Loboda, 2001). In the case of the open Shaker channel, because of a very large $k_{\text {off }}$, the $K d(0 \mathrm{mV})$ is quite large, $57 \mathrm{mM}$, and thus open Kv4.2 and Shaker channels share the property of a low affinity for 4AP. These values for the $k_{o n}$ for open channels contrast sharply with those reported for closed state block. In $\mathrm{Kv} 4.2$ at $-80 \mathrm{mV}$, the $k_{\text {on }}$ of $80 \mathrm{M}^{-1} \mathrm{~s}^{-1}$ (Fig. 4C) compares well to the value of $100 \mathrm{M}^{-1} \mathrm{~s}^{-1}$ for the binding to closed Kv3.1 channels (Kirsch and Drewe, 1993). In Kv3.1 channels, which demonstrate both closed and open channel block, the $k_{o n}$ for closed channels is roughly 200 -fold smaller than for open channels. Given that the concentration-block relationship for the block of Kv3.1, as in other $\mathrm{Kv}$ channels (McCormack et al., 1994) and in this study (Fig. 2), is consistent with a 1:1 binding model, Kirsch and Drewe (1993) proposed that there is a single binding site (as opposed, for example, to a site on each of the four $\alpha$-subunits) on the cytoplasmic side of the conduction pathway and that the difference in the rates of closed and open channel block simply reflected differing accessibilities to that site. A possible explanation for the divergence of $k_{o n}$ for open and closed channel block is guarding of the binding site by the activation gate, which is formed by the convergence of S6 segments on the cytoplasmic side of the pore. In Shaker, Kv3.1 and Kv2.1 channels with an open activation gate, $4 \mathrm{AP}$ has an on-rate that approaches that for pore blockers, such as $\mathrm{TEA}^{+}$, and it is conceivable that the very small $k_{o n}$ measured at rest in $\mathrm{Kv} 3.1$ and in $\mathrm{Kv} 4.2$ is due to a very small open probability at rest. 
The 4AP block of Kv4.2 could be modeled either by assuming slow unbinding of 4AP from open bound channels, as proposed for the block of the SGA delayed rectifier and Kv3.1 channels (Yeh et al., 1976a; Kirsch and Drewe, 1993), or that 4AP stabilized the preopen closed state such that the rate-limiting step of channel opening preceded rapid channel unblock (Armstrong and Loboda, 2001). The latter mechanism was simulated in Scheme 4AP (not shown) by decreasing the rate of the $\mathrm{C}_{4} \mathrm{~B}$ to $\mathrm{OB}$ transition from 400 to $18 \mathrm{~s}^{-1}$ and destabilizing the open state by increasing the rate of the $\mathrm{OB}$ to $\mathrm{C}_{4} \mathrm{~B}$ transition from 1100 to $3544 \mathrm{~s}^{-1}$; once open, the off-rate of $4 \mathrm{AP}$ was assumed to be $10^{5} \mathrm{~s}^{-1}$; however, because the $P_{o}$ of closed-bound states would decrease relative to closed unbound channels, an expected consequence of this gating scheme is that recovery from resting block would acquire some degree of use dependence. This remains to be definitively studied using fast application of 4AP to inside-out patch recordings. At this time, a mechanism of action similar to that proposed for Shaker channels (Armstrong and Loboda, 2001) cannot be ruled out and indeed has some appeal given that CSI has been proposed to involve a dissociation or slippage between voltage sensor movement and activation gate opening (Dougherty et al., 2008; Bähring and Covarrubias, 2011). On the other hand, the finding that $k_{\text {on }}$ in $\mathrm{Kv} 4.2$ is "independent of the membrane voltage in the range of -80 to $-120 \mathrm{mV}$ " (Tseng et al., 1996) is a plausible argument against a dependence of tonic block on a small $P_{o}$ at rest. Jerng and Gilly (2002) have also expressed reservations about ascribing the potent resting block of the giant fiber lobe delayed rectifier current versus the virtual absence of resting block in sqKv1A to a difference in the resting $P_{o}$.

In summary, the binding of $4 \mathrm{AP}$ to closed channels causes a concentration-dependent block of $\mathrm{Kv} 4.2$ currents that can manifest during a depolarizing step as an apparent slowing of activation and a delayed onset of inactivation that can cause the test current to cross over the control current. 4AP appears to have a very low or no affinity for open channels, and 4AP binding and inactivation are mutually exclusive processes. These features are replicated in a gating scheme in which 4AP is assumed to have no effect on gating current and in which there is either slow unbinding from open channels or a biasing of the concerted opening/closing step more strongly to the preopen closed state. Whether the small $k_{o n}$ for closed-state block of Kv4.2 channels is due to a low open probability at rest remains an unanswered question.

\section{Acknowledgments}

Thanks to Zhuren Wang for expert technical assistance.

\section{Authorship Contributions}

Participated in research design: Kehl.

Conducted experiments: Kehl.

Contributed analytic tools: Kehl.

Performed data analysis: Kehl.

Wrote or contributed to the writing of the manuscript: Kehl.

\section{References}

Armstrong CM (1971) Interaction of tetraethylammonium ion derivatives with the potassium channels of giant axons. J Gen Physiol 58:413-437.

Armstrong CM (1997) A closer picture of the $\mathrm{K}$ channel gate from ion trapping experiments. J Gen Physiol 109:523-524.
Armstrong CM and Loboda A (2001) A model for 4-aminopyridine action on $\mathrm{K}$ channels: similarities to tetraethylammonium ion action. Biophys $J \mathbf{8 1}$ : 895-904

Bähring R, Boland LM, Varghese A, Gebauer M, and Pongs O (2001) Kinetic analysis of open- and closed-state inactivation transitions in human Kv4.2 A-type potassium channels. $J$ Physiol 535:65-81.

Bähring R and Covarrubias M (2011) Mechanisms of closed-state inactivation in voltage-gated ion channels. J Physiol 589:461-479.

Birnbaum SG, Varga AW, Yuan LL, Anderson AE, Sweatt JD, and Schrader LA (2004) Structure and function of Kv4-family transient potassium channels. Physio Rev 84:803-833.

Campbell DL, Qu Y, Rasmusson RL, and Strauss HC (1993) The calciumindependent transient outward potassium current in isolated ferret right ventricular myocytes. II. Closed state reverse use-dependent block by 4 -aminopyridine. $J$ Gen Physiol 101:603-626.

Castle NA, Fadous S, Logothetis DE, and Wang GK (1994a) Aminopyridine block of Kv1.1 potassium channels expressed in mammalian cells and Xenopus oocytes. Mol Pharmacol 45:1242-1252.

Castle NA, Fadous SR, Logothetis DE, and Wang GK (1994b) 4-Aminopyridine binding and slow inactivation are mutually exclusive in rat Kv1.1 and Shaker potassium channels. Mol Pharmacol 46:1175-1181.

Castle NA and Slawsky MT (1993) Characterization of 4-aminopyridine block of the transient outward $\mathrm{K}^{+}$current in adult rat ventricular myocytes. J Pharmacol Exp Ther 265:1450-1459.

Cheng YM, Fedida D, and Kehl SJ (2010) Kinetic analysis of the effects of $\mathrm{H}^{+}$or $\mathrm{Ni}^{2+}$ on Kv1.5 current shows that both ions enhance slow inactivation and induce resting inactivation. J Physiol 588:3011-3030.

Colquhoun D and Hawkes AG (2011) A Q-matrix cookbook, in Single Channel Recording, pp 589-633, Plenum Press, New York.

Dougherty K, De Santiago-Castillo JA, and Covarrubias M (2008) Gating charge immobilization in Kv4.2 channels: the basis of closed-state inactivation. J Gen Physiol 131:257-273.

Doyle DA, Morais Cabral J, Pfuetzner RA, Kuo A, Gulbis JM, Cohen SL, Chait BT, and MacKinnon R (1998) The structure of the potassium channel: molecular basis of $\mathrm{K}^{+}$conduction and selectivity. Science 280:69-77.

Fiset C, Clark RB, Shimoni Y, and Giles WR (1997) Shal-type channels contribute to the $\mathrm{Ca}^{2+}$-independent transient outward $\mathrm{K}^{+}$current in rat ventricle. $J$ Physiol 500: $51-64$

Jerng $\mathrm{HH}$ and Covarrubias M (1997) $\mathrm{K}^{+}$channel inactivation mediated by the concerted action of the cytoplasmic N- and C-terminal domains. Biophys J 72:163-174.

Jerng HH and Gilly WF (2002) Inactivation and pharmacological properties of sqKv1A homotetramers in Xenopus oocytes cannot account for behavior of the squid "delayed rectifier" $\mathrm{K}^{+}$conductance. Biophys $J$ 82:3022-3036.

Kehl SJ (1990) 4-Aminopyridine causes a voltage-dependent block of the transient outward $\mathrm{K}^{+}$current in rat melanotrophs. J Physiol 431:515-528.

Kehl SJ, Fedida D, and Wang Z (2013) External $\mathrm{Ba}^{2+}$ block of Kv4.2 channels is enhanced in the closed-inactivated state. Am J Physiol Cell Physiol 304: C370-C381.

Kirsch GE and Drewe JA (1993) Gating-dependent mechanism of 4-aminopyridine block in two related potassium channels. J Gen Physiol 102:797-816.

Kirsch GE and Narahashi T (1983) Site of action and active form of aminopyridines in squid axon membranes. J Pharmacol Exp Ther 226:174-179.

Kirsch GE, Yeh JZ, and Oxford GS (1986) Modulation of aminopyridine block of potassium currents in squid axon. Biophys $J$ 50:637-644.

Loboda A and Armstrong CM (2001) Resolving the gating charge movement associated with late transitions in $\mathrm{K}$ channel activation. Biophys $J$ 81:905-916.

McCormack K, Joiner WJ, and Heinemann SH (1994) A characterization of the activating structural rearrangements in voltage-dependent Shaker $\mathrm{K}^{+}$channels. Neuron 12:301-315.

Niwa N and Nerbonne JM (2010) Molecular determinants of cardiac transient outward potassium current (I(to)) expression and regulation. J Mol Cell Cardiol 48: $12-25$.

Rosenthal JJ, Vickery RG, and Gilly WF (1996) Molecular identification of SqKv1A: a candidate for the delayed rectifier $\mathrm{K}$ channel in squid giant axon. $J$ Gen Physiol 108:207-219.

Spires S and Begenisich T (1989) Pharmacological and kinetic analysis of K channel gating currents. J Gen Physiol 93:263-283.

Thompson S (1982) Aminopyridine block of transient potassium current. J Gen Physiol 80:1-18.

Thompson SH (1977) Three pharmacologically distinct potassium channels in molluscan neurones. J Physiol 265:465-488.

Tseng GN, Jiang M, and Yao JA (1996) Reverse use dependence of Kv4.2 blockade by 4-aminopyridine. J Pharmacol Exp Ther 279:865-876.

Yeh JZ, Oxford GS, Wu CH, and Narahashi T (1976a) Dynamics of aminopyridine block of potassium channels in squid axon membrane. $J$ Gen Physiol 68:519-535.

Yeh JZ, Oxford GS, Wu CH, and Narahashi T (1976b) Interactions of aminopyridines with potassium channels of squid axon membranes. Biophys $J$ 16:77-81.

Yeola SW and Snyders DJ (1997) Electrophysiological and pharmacological correspondence between $\mathrm{Kv} 4.2$ current and rat cardiac transient outward current. Cardiovasc Res 33:540-547.

Address correspondence to: Steven J. Kehl, Department of Cellular and Physiological Sciences, University of British Columbia, 2350 Health Sciences Mall, Vancouver, BC, Canada V6T 1Z3. E-mail: skehl@mail.ubc.ca 